\title{
Identifying unfamiliar voices: the influence of sample duration and parade size.
}

\author{
Nikolas Pautz ${ }^{\mathrm{a}}$, Harriet M. J. Smith ${ }^{\mathrm{a}}$, Katrin Mueller-Johnson ${ }^{\mathrm{c}}$, Francis Nolan ${ }^{\mathrm{b}}$, Alice Paver $^{\mathrm{b}}$, \\ Kirsty McDougall ${ }^{\mathrm{b}}$ \\ ${ }^{a}$ Department of Psychology, Nottingham Trent University \\ ${ }^{\mathrm{b}}$ Theoretical and Applied Linguistics Section, Faculty of Modern and Medieval \\ Languages and Linguistics, University of Cambridge \\ ${ }^{\mathrm{c}}$ Centre for Criminology, University of Oxford
}

\section{THIS PAPER HAS NOT BEEN PEER-REVIEWED}

Correspondence concerning this article should be addressed to Nikolas Pautz, Department of Psychology, Nottingham Trent University, 50 Shakespeare Street, Nottingham, NG1 4FQ. Email: nikolas.pautz@ntu.ac.uk

OR

Harriet Smith, Department of Psychology, Nottingham Trent University, 50 Shakespeare Street, Nottingham, NG1 4FQ. Email: harriet.smith02@ntu.ac.uk

This work was supported by an Economic and Social Research Council (ES/S015965/1) grant awarded to Kirsty McDougall. 


\begin{abstract}
Objectives: Voice identification parades can be unreliable due to the error-prone nature of earwitness responses. In England and Wales, Home Office guidelines (2003) recommend that voice parades should consist of nine-voices, each played for 60 s. This makes parades resourceconsuming to construct. In the present paper we conducted two experiments to see if voice parade procedures could be simplified. In Experiment 1, we compared identification performance between $15 \mathrm{~s}, 30 \mathrm{~s}$, and 60 s voice samples in nine-voice parades where the target was present or absent. In Experiment 2, we explored if the same sample duration conditions used in Experiment 1 would lead to different outcomes if a six-voice parade were used. We also pooled the data from both experiments to see if performance differed between early and late target positions in target-present parades.
\end{abstract}

Hypotheses: For Experiment 1, we hypothesised that the reduced relative distance between voice samples and pauses facilitated by shorter sample duration would offset any advantages of the increased amount of identity information afforded by longer sample duration times. For Experiment 2, we hypothesised that fewer voices in a parade would reduce auditory attentional demands and subsequently reduce the propensity for erroneous comparisons with the encoded voice of the 'perpetrator'.

Method: Both Experiment $1(\mathrm{~N}=271,135 \mathrm{~F})$ and Experiment $2(\mathrm{~N}=270,136 \mathrm{~F})$ were conducted online using Gorilla and participants recruited from Prolific. Experiments were between-subjects designs and stimuli were taken from forensically-oriented speech databases. 
Results: The results show that accuracy and signal sensitivity is similar when using the 15- and 60-second voice samples regardless of the whether six or nine voices are used in the parade. However, there was converging evidence that the advantages of either a short or a long voice sample are not additive when trying to reach a middle-ground of 30s. Performance on targetabsent parades - which simulate an innocent suspect being apprehended - were at chance-levels in both parade sizes and response criteria were mostly liberal, indicating a strong propensity-tochoose effect.

Conclusions: We provide initial empirical evidence supporting the proposal that duration of voices in parades can be limited to $15 \mathrm{~s}$ without adversely affecting identification performance. We also argue that more work is required to address the issue of high false alarm rates before any reduction in parade size can be considered.

Keywords: earwitness, voice parades, voice identification, witness memory, system variables 


\section{Introduction}

During some crimes the perpetrator is heard, but not seen. The severity of such crimes might vary from attempted telephone fraud to crimes such as rape or murder where disguises may be worn ( $R v$ Khan and Bains, 2002, discussed in Nolan, 2003). If the only identification evidence linking the perpetrator to the crime is the sound of their voice, this evidence might be decisive in court (Robson, 2016). In these situations, the person who overheard the perpetrator's voice during the crime is often referred to as an 'earwitness'. Earwitnesses may be asked by the police to try to identify the voice of a perpetrator from a voice parade.

In England and Wales, Home Office (2003) guidelines provide recommendations about how a voice parade should be constructed. The guidelines were innovative at the time of their development in that they promoted an approach to voice sample construction that was underlined with a research-based phonetic understanding of speech. However, despite the utility of certain aspects of the guidelines, the procedural recommendations are not evidence-based, and, importantly, were adapted from face identification procedures ${ }^{1}$ rather than being designed specifically for voices (Smith et al., 2020). This paper builds on the work done by Smith et al. (2020) in proposing amendments to the Home Office (2003) voice parade procedures through improved understanding of system variables (Wells, 1978), i.e., variables that are controllable by law enforcement. The conclusions of this body of research are relevant to all jurisdictions where earwitness evidence is admitted as evidence; this includes much of Europe, the US, Australia, and Canada (Broeders \& van Amelsvoort, 2001; Cantone, 2010; Laub et al., 2013; McGorrery \&

\footnotetext{
${ }^{1}$ [author name removed for purposes of blinded peer review] notes that at the time the police were in favour of making the procedural aspects as close as possible to the visual procedure to minimise the opportunity for challenge by the defence.
} 
McMahon, 2017). The present paper focuses on providing evidence-based recommendations for the system variables of parade size and the voice sample duration. The Home Office guidelines recommend that "a total of nine samples should be selected (i.e., the suspect's plus eight others)", and that "these [samples] should each be about one minute long..." (Home Office, 2003, point 13). There are both practical reasons and theoretical reasons for attempting to manipulate both the number and length of the voice samples included in a voice parade, which we summarise below. We provide an overview of the current and historical limitations of earwitness research and outline how the present study attempts to overcome some of these.

\section{Sample duration}

In the UK, constructing a voice parade involves several stages. First, an expert phonetician must pick out similar-sounding suspects from recorded police interviews to act as foils. The expert selects excerpts of speech from the recordings, but the content must be unrelated to the crime in question, and contain no identifying information. They then splice together the speech excerpts to form voice samples for both the foils and the suspect (see Nolan, 2003, for a detailed description of the procedure forensic phoneticians use to produce the voice samples). This complicated and time-consuming process may increase the delay between the crime (encoding) and the parade (retrieval). Prolonged retention intervals risk reducing the strength of the initial memory (of overhearing the perpetrator's voice) and make successful identification less likely (King et al., 2002). A specific practical reason for the delay in preparation is that it can be difficult to locate enough suitable recordings of voices to produce 60s samples. Simply put, by reducing the duration of the voice samples, it would in, in general, take less time to source material and to splice together the samples to be used in the parade. 
While the 60s sample duration recommendation by the Home Office seems like a reasonable amount of time needed for a listener to build a representation of a voice in order to compare it to their memory of the perpetrator, people can extract basic information from voices after less than a second of exposure, such as emotion (Bestelmeyer et al., 2010) and aspects of personality (McAleer et al., 2014). While unfamiliar voice identification would likely require somewhat longer exposure, it does mean that the 60 s voice sample may be unnecessarily long if it offers no advantage over shorter durations. The only research that has focused on addressing the effect of sample duration was conducted by Smith et al. (2020). They compared 15s and 30s samples and found that there was no difference in accuracy, arguing that this lack of effect may have been because the amount of identity information in 15 - and 30s samples did not differ to the extent to which it activated the auditory representation of the target voice. Additionally, the authors suggested that even if there was a difference in the amount of identity information between the two sample durations, the benefits were offset by the additional interference associated with longer sample durations. A limitation of the Smith et al. (2020) study, however, was that comparisons were not made against the Home Office guidelines recommending 60s samples, meaning that the research cannot be used to support procedural recommendations. It is important to highlight that a shorter duration does not necessarily need to result in higher accuracy for changes to be recommended; if the shorter duration is not worse than a longer duration, the practical utility of a shorter duration is clear.

In addition to the practical benefits of reducing the sample duration of the voices included in a voice parade, there are theoretical reasons to believe that a shorter duration may be beneficial, or at the very least not harmful to performance. Temporal-ratio models of memory suggest that time is an important dimension which underpins memory organization and retrieval 
(Crowder, 2014; Bjork \& Whitten, 1974; Brown et al., 2007). The basis of temporal models of memory is that more recent items are more easily discriminable from distant items. Bjork and Whitten (1974) proposed a ratio-like mechanism, arguing that the distinctiveness of previously encoded items is determined by the "ratio of the temporal separation of successive items to the temporal delay from those items to the point of recall” (p. 189). In other words, the further away the items are from the point of recall, the greater the separation between the memories needs to be in order for the memories to be distinct (Crowder, 2014).

It is possible that, in the context of voice identification parades, the relative temporal distance between the voices - i.e., the pauses between the speech samples - may act as such 'separators' breaking up the samples into distinct identity percepts. This suggests that the voice samples in parades using shorter sample durations should be more distinct than those using longer sample durations as they are more likely to be closer to the optimal temporal ratio, even if they do not meet it entirely. In other words, the optimal temporal pause for shorter voice samples would be substantially shorter than that required for longer voices; this closer-to-optimal characteristic would facilitate increased voice distinctiveness. More distinctive identity percepts are easier to compare both with each other (a relative judgement) and with the original voice (an absolute judgement). Thus, because longer samples are not necessarily needed to form stable identity percepts (Bestelmeyer et al., 2010; McAleer et al., 2014) and successive memorization requires ever-increasing pause times (Crowder, 2014), longer voices may end up being harmful in terms of memory distinctiveness in the context of voice parades.

It is helpful to frame this in terms of Signal Detection Theory (SDT; Macmillan \& Creelman, 2004), which has emerged as a powerful framework for comparing the strength of witness memory accuracy using different types of identification procedures (Cohen et al., 2020). 
Signal detection models provide two metrics of interest. The first of these is the response criterion, which in the context of identification parades measures the participants' willingness to respond that the signal (i.e., the target voice) is present independently of the information that the participants received. The second metric, which is of primary interest to the present paper, is signal sensitivity; signal sensitivity ( $d$ prime) is a measure of how well a listener can detect a signal amongst noise (DeCarlo, 1998). If the theoretical premise discussed above holds true, the increased distinctiveness of shorter samples may facilitate the ability of listeners to discriminate the signal (which would be the sample of the perpetrator's voice in the parade) from the noise (which would be the similarly sounding foil voice samples) within a voice parade.

\section{Size of the parade}

Just like sample duration, the size of the parade (i.e., the number of speakers in the parade), has both practical and potentially theoretical implications. The practical implications of using fewer voices are similar to that of using shorter sample durations: the resources required to set up a voice parade can all be reduced if there are fewer foils. This would ultimately lead to a shorter interval between exposure and identification, reducing the risk of memory decay.

The number of foils used in previous voice identification research has varied. Lab-based voice parades have commonly used either five (Cook \& Wilding, 1997b, 1997a; Kerstholt et al., 2006; Smith, \& Baguley, 2014) or eight (McDougall et al., 2015; Smith et al., 2020), though there are some exceptions (for example, Ohman et al., 2011, used six foils). An important benefit of a larger parade is that the additional foils offer an innocent suspect increased protection. For instance, if target-absent performance is at chance level (e.g., Sherrin, 2015) an innocent suspect 
has a ten percent chance of being randomly selected ${ }^{2}$ if they are included in a nine-voice parade compared with over a 14 percent chance in a six-voice parade. However, there have been few psychological studies which have experimentally tested the ideal number of foils for voice parades. One of these was a study was undertaken by Bull and Clifford (1984) who compared voice parades with four, six, and eight voices and concluded that a six is the optimal number with performance dropping when parades are either longer or shorter. In contrast, a study by Hammersley and Read (1996) concluded that 20 voices is the optimal number to protect an innocent suspect. Both studies could be considered exploratory, and both had limitations: for instance, Bull and Clifford (1984) did not take into account the protection afforded to potentially innocent suspects when more foils are included, while Hammersley and Read (1996) did not take into account the resources that would be required to build 20-voice parades. Additional research is required to provide any type of evidenced-based policy recommendation regarding the number of foils used in a voice parade.

In terms of theoretical implications, listening to a series of voices in a parade requires attention. The longer and more numerous the voices, the greater the attentional resources required. Zimmerman and colleagues (2016) posit that auditory attention, like all cognitive processes, can be faulty, but that the propensity for erroneous auditory attention is likely to be linked to high resource demand. The authors suggest that there is a greater risk of erroneous processing when an incoming signal is compared to information already stored in long-term memory. As noted previously, comparing incoming signals (the voice samples) to information

\footnotetext{
${ }^{2}$ An earwitness undertaking a parade with ninevoices will have ten possible options to choose from - one of the nine voices or none of the voices - but they can only select one of these options. If a listener were to randomly select an option, they would have a tenpercent chance of selecting the suspect if the suspect is included as well as a tenpercent chance of correctly selecting that the target was not present in a target-absent condition.
} 
already stored in long-term memory (the perpetrator's voice) is the essence of a voice identification parade.

In terms of SDT, this suggests that signal sensitivity strength may be negatively impacted by the attentional resource requirements of the voice parade. If this holds true, reducing the number of voices, and thus attentional demands, may limit the risk of erroneous comparisons and increase signal strength. In addition to auditory attention, by reducing the total number of voices to review in the parade, there may be, as per temporal-ratio models of memory (Bjork and Whitten, 1974; Brown et al., 2007), a reducing in the optimal temporal distance between the least and most recent voices in the parade, facilitating both relative and absolute judgements.

\section{Confidence}

Research using unfamiliar voices often shows unreliable confidence-accuracy relationships, ranging from weak or null relationships (Smith et al., 2020; Kerstholt et al., 2004; Öhman et al., 2011; Olsson et al., 1998) to significant relationships (Yarmey, 1991; Bull \& Clifford, 1984; Saslove \& Yarmey, 1980). Despite this unreliable relationship, self-reported confidence has been found to influence mock jurors judging the reliability of witness voice identification (Van Wallendael et al., 1994). Additionally, previous research has suggested that a witness' confidence in their judgement varies based on the perceived difficulty of the parade (Olsson, 2000). Thus, it is important to consider the potential effects of any procedural changes on listener confidence.

\section{Limitations of earwitness research}

Apart from the dearth of research into system variables, psychological investigations into voice identification tend to exhibit limitations that the present research attempts to rectify. First, 
earwitness research in the psychological literature generally uses only one or two targets when designing the parades (e.g., McAllister et al., 1993; Öhman et al., 2013a; Philippon et al., 2013). This does little to alleviate concerns about whether any results are influenced by a particularly distinct target, which may affect not only the external validity of the results, but the construct validity, too (Wells \& Windschitl, 1999). Research suggests that a distinctive voice is more likely to be correctly identified regardless of familiarity (Skuk \& Schweinberger, 2013; Stevenage, 2018; Yarmey, 1991). This highlights the importance of the foil selection method to ensure that a fair parade is developed.

Voice parades in experimental research are often constructed relatively arbitrarily and without consideration of similarity relative to the target. While some research has used description-match and suspect-match (Tunnicliff \& Clark, 2000; Wells, 1993) methods to select parade foil samples (Cook \& Wilding, 1997b; Kerstholt et al., 2004), the average persons' available vocabulary to define the characteristics of a voice is far more limited than that which can be used to define a face (see Kelly, 2018). Thus, the efficacy of these match-approaches may be limited for voice parades. A strength of the present research is the use of multi-dimensional scaling methods to develop quantitatively fair parades from established forensic voice databases (detailed in Appendix A). This is the approach used by forensic phoneticians when constructing 'real' voice parades (see McDougall, 2013, for more information). Thus, by adopting this approach, we maximise the ecological validity of the experiments.

We argue that the very nature of developing a voice parade requires an interdisciplinary approach. To the best of our knowledge, no previous psychology studies have taken such an approach. It is often experts in disciplines other than psychology, such as phonetics and linguistics, who are asked to assist with the preparation of voice parade samples in real cases. To 
determine the most effective ways to conduct a voice parade, expertise and research understanding in speech behaviour and speaker variation, psychological research on memory and witness behaviour, and input from criminology concerning police produce and interaction, need to be drawn on in concert. The present research has benefited from such an approach, from conceptualization and design, to interpretation.

Finally, the analytical procedures used to derive results and infer interpretations often do not take into account the full utility of the data. For example, Wixted et al. (2016) argues that by using alternative analytical procedures, such as SDT models as opposed to simpler regression models, we can get a more nuanced interpretation of the same data. In the present studye combine both traditional binary analyses with SDT models which allow us to go beyond measuring accuracy and to provide metrics of decision criteria and sensitivity. However, as SDT models have not been widely used in the psychological earwitness literature, direct comparisons between results of the present research and prior studies are limited.

\section{The current study}

The research presented in this paper aims to provide clarity on two system variables that are fundamental to the construction of any voice parade: the duration of the voice samples and the number of foil voices included. We present the results of two experiments that followed the Home Office (2003) procedure as closely as possible. While the primary objective of the present research is to investigate whether sample duration and parade size influence accuracy, signal sensitivity, and response criterion, we also look at the relationship between confidence and accuracy.

\section{Experiment 1}


In Experiment 1, we investigated whether reducing the sample duration to 30s or $15 \mathrm{~s}$ improves witness performance (i.e., a listener's ability to distinguish between the signal [the target] and noise [the foils]) compared to 60s samples in a nine-voice parade. Based on temporalratio models of memory (Bjork \& Whitten, 1974; Brown et al., 2007), we hypothesised that the reduced relative distance between voice samples and pauses facilitated by shorter sample duration times, as well as the reduced attentional demands, will offset any advantages of the increased amount of identity information afforded by longer sample duration times. Specifically, we hypothesised that overall accuracy rates and signal sensitivity will not differ meaningfully across sample durations.

\section{Method}

\section{Participants $^{3}$}

A total of $\mathrm{N}=277$ participants were recruited via the online recruitment platform Prolific.co. Six participants were excluded (five for having average between-voice response times (RT) that were clear outliers, and one due to non-completion of the filler task) making the final sample size $\mathrm{N}=271$. The average age of the participants was 27.68 years old $(S D=6.16)$ with 136 males and 135 females. Participants were required to (1) have been born in England; (2) have lived most of their life before turning 18 in England; (3) speak English as their first language; (4) have no uncorrected hearing loss or hearing difficulties; (5) be between the ages of

\footnotetext{
${ }^{3}$ The sample size was justified by balancing heuristic norms and resources constraints (Lakens, 2021). In terms of the heuristic norms, the sample size used here is either on par with, or exceeds, those used in previous voice identification research (e.g., Perfect et al., 2002; Kerstholt et al., 2006; Philippon et al., 2013; Smith et al., 2020). The present research was also constrained by the cost of participant recruitment. Unlike eyewitness identification research which allows for the possibility of each participant completing multiple parades and therefore providing multiple data points (Mansour et al., 2017), earwitness identification research is often limited to a single data point per participant (largely due to interference negatively affecting auditory memory for voices, see Stevenage et al., 2011).
} 
18 and $40^{4}$. Additionally, participants were required to have a minimum approval rate of $90 \%$ on

Prolific and a minimum internet connection speed of over $5 \mathrm{mb} / \mathrm{s}$. Ethical approval was granted by the Research Ethics Committees of [BLINDED] and [BLINDED].

\section{Design}

Experiment 1 employed a between-subjects 3 (sample duration: 15s, 30s, or 60s) x 2 (target: present or absent) factorial design.

\section{Apparatus and materials}

The speech materials used in this study were taken from the Dynamic Variability in Speech Database (DyViS) (Nolan et al., 2009; available to download from the UK Data Service), the York Variation in Speech database (YorViS) (McDougall et al., 2015; contact author for permission), and the West Yorkshire Regional English Database (WYRED; Gold et al., 2018, available from the UK Data Service). Further details can be found in Appendix A, Table A1.

DyViS, YorViS and WYRED each contain recordings of a relatively large number of speakers sharing the same demographic characteristics, i.e., speakers of the same age (18-30 years), sex (male) and accent background (DyViS: SSBE, YorViS: York English, WYRED: Bradford/Kirklees/Wakefield English), making them ideal sources of speech recordings for constructing ecologically valid voice parades. The demographic control offered by these databases enables the researcher to choose appropriate foils for comparison with a suspect as is essential in a real parade context.

\footnotetext{
${ }^{4}$ This eligibility criterion was implemented to minimize the chance of participants having age-related hearing decay (Hoffman et al., 2017).
} 
All databases used the same elicitation techniques. There were two tasks relevant to the current research. One task, used for the encoding sample, was a mock telephone call between a speaker (the 'perpetrator') and an accomplice who was on the other end of the phone, and whose speech was not audible. The participant who was speaking into the telephone in the studio, and being recorded at studio quality in situ, was also recorded at the remote end of the telephone line. The other task, used for the parade samples, was a simulated police interview designed to elicit spontaneous speech in a situation of cognitive conflict (i.e., lying). The speaker plays the role of a suspect being interviewed. In both tasks, the speech produced by the participant is not scripted and relatively spontaneous in style. The participant responds to questions from the accomplice (phone call) or interviewer (police interview), guided by visual information containing names of characters, streets, locations, etc.

Speaker selection. To construct the parades a total of six groups comprising ten speakers each (one target, one replacement, and eight foils) were selected following multidimensional scaling analysis (see Appendix A for a detailed overview of this process). Three of these groups used SSBE speakers from the DyViS database, two used speakers from the WYRED database (one Wakefield English group, one Bradford English group), and the last featured speakers of York English from the YorViS database. These speaker groups involving four accents were purposely selected in order to highlight possible accent-specific effects that would not be clear if only a single accent was used. Further, three SSBE groups were included to enable examination of variation in individual targets within a single accent.

Encoding samples. The telephone task encoding samples were all approximately $60 \mathrm{~s}$ in duration and were taken from the telephone conversation with the accomplice. Participants did not hear the target's interlocutor. These samples were used to simulate the participant (i.e. 
'earwitness') overhearing a crime. Pauses where the interlocutor on the other end of the phone was speaking were cut so that the net speech was similar for all encoding samples. All clips had to be clearly audible and hesitations that were particularly salient in terms of their length were avoided.

Parade samples. All the voice samples used in the parade were taken from the studioquality simulated police interviews to replicate the process used by the police. Excerpts featuring the interviewees were spliced together to produce 15,30 , and 60 s samples. The voice samples were taken from different sections of each interview so that the content of speech differed across speakers ${ }^{5}$. For both the encoding and parade samples, one second of silence, taken from a silent section of each respective interview, was inserted between utterances, and any extraneous noises (coughs, sneezes, chairs moving) and speech of the interlocutor were removed. Furthermore, the content of the interview samples did not overlap with the content of the phone recording. All audio stimuli were normalized for intensity. The order of the excerpts was randomised to ensure that there was no continuous narrative within each sample.

\section{Procedure}

Overview. The experiment was hosted on the online experiment builder platform Gorilla.sc (Anwyl-Irvine et al., 2020). Participants were instructed to complete the experiment in a quiet environment with no distractions. Participants were required to calibrate their headphone volume and then undertook a headphone screening assessment (Woods et al., 2017) to ensure they were using headphones or earphones. Participants were allowed two attempts to identify

\footnotetext{
${ }^{5}$ It is not possible for the content of the recordings to be the same across parade members as, in the same way as with a real parade, the excerpts are taken from a recording of spontaneous speech. However, the greatest effort was taken to ensure that the content of the excerpts was as innocuous as possible, containing no information specifically relating to the fictional crime being discussed in the original recordings.
} 
correctly at least $4 / 6$ of the 'softest' tones - if they failed both attempts, they were rejected from the experiment.

As participants progressed through the experiment, there was no option to go back and review previous elements. Upon successful completion of the headphone screening task, the participants were randomly allocated to target-speaker groups (DyViS 1, 2, 3, WYRED 1, 2, and YorViS), condition (15s, 30s, 60s samples), and type of parade (target-present or target-absent) using balanced randomisation. Each participant completed a single trial. They were not told that they would have to complete a voice parade, but rather that they would be undertaking an experiment on voice perception. This facilitated incidental as opposed to intentional encoding of the voice. This is important as witnesses are, arguably, more likely to be focusing on the event (incidental) rather than trying to remember the details so that they can be recalled at a later point in time (intentional) (Smith et al., 2020). In target-present parades, the target voice was either in a relatively early (third) or late (seventh) position to counterbalance for positional effects.

Phase 1 - Encoding. Participants listened to the 60s encoding sample when they were ready to begin the experiment by clicking a 'Play' button on the screen. Participants automatically moved on to the next stage of the experiment when the encoding sample finished playing.

Phase 2: Storage. After listening to the encoding sample, participants completed a filler task. This task comprised a wordsearch (of different fruit names) with numbered axes. Participants were instructed to find as many words as possible and list the $\mathrm{X}$ and $\mathrm{Y}$ axis numbers of the first letter of each word. At the same time, they listened to a recording of ambient noise, which was made in a public lobby and featured unintelligible speech sounds. A five-minute timer 
countdown was displayed in the top right corner of the screen. We excluded participants who entered less than five words in the filler task unless an explanation was provided in the debrief (e.g., they were not sure how to 'enter in' a word).

Phase 3 - Retrieval. Before the parade began, participants read that they would be listening to a voice parade which contained 'a series of nine voices'. Participants were asked to 'imagine that the voice you heard initially was one that you overheard in a public setting where you could hear but not see the speaker'. Importantly, participants were told, 'the speaker may or may not be present in the parade', and if they felt that the speaker was absent, they should indicate this by selecting the option of 'none' when asked 'which voice do you think belonged to the perpetrator?'. Having read the pre-parade instructions, the voice parade began immediately once participants had clicked 'continue'. Each voice sample in the parade corresponded with a voice number (Voice 1, Voice 2, etc.). To encourage participants to reorient their attention prior to hearing each voice sample, they pressed the spacebar in order to proceed to the next voice. The recorded RTs enabled us to check that the participants were paying attention to the task (for both experiments; Mean $R T=10898 \mathrm{~ms} ; S D=7319 \mathrm{~ms})$.

After listening to all nine voices in the parade, participants selected which voice, if any, they thought belonged to the perpetrator. On this page, participants were reminded to select 'none' if they thought the target speaker was absent. After registering their decision, participants rated their confidence in their decision using a 11-point slider scale, ranging from 0 (not at all confident) to 10 (extremely confident).

Participants completed a series of debrief questions after finishing the experiment. Responses to these were inspected but not formally analysed. Included in these were open-ended 
questions about the strategy used to identify the perpetrator, whether they considered responding that the perpetrator was not present, and if any technical difficulties were experienced. We also included a five-point Likert scale asking participants to rate if they thought the parade was very easy (1) up to very difficult (5). On the post-parade debriefing question (eliciting 1-5 Likert scale responses), descriptively speaking, participants found the target-absent parade to be, on average, slightly more difficult than the target-present parade (target-absent: Mean=3.78, $S D=0.99$; targetpresent: Mean=3.51, $S D=1.09$ ).

\section{Results}

\section{Accuracy}

Data were analysed using Bayesian mixed models (Gelman et al., 2014; McElreath, 2016) with accurate parade identifications scored as 1 and inaccurate identifications as 0 , in a 3 (sample duration: 15s, 30s, 60s) x 2 (target presence: present or absent) factorial design. This analysis treated the six targets as a random factor. The 60s sample duration condition was treated as the reference category. Leave-one-out cross validation was used to evaluate model comparisons (Vehtari et al., 2017). The fitted models' predictive performance was estimated as the sum of the expected log pointwise predictive density $(\widehat{e l p d})$ ) alongside its standard error (SE). A model with a difference in $\mathrm{SE}(\triangle \mathrm{SE})$ equal to or greater than 5 is suggestive of statistically better performance (Vehtari et al., 2017). While the model with only target presence included as a predictor resulted in the highest predictive performance, no model exceeded a $\Delta \mathrm{SE}$ of 5. Thus, we selected the model including interactions for inference (for the full model, see Appendix B, Table B2). 
The results of the model indicate that there is negligible ${ }^{6}$ evidence to support the hypothesis that accuracy in the $15 \mathrm{~s}$ and 30 s sample conditions differed meaningfully from accuracy in the 60 s sample condition. While there was moderate evidence to support the hypothesis that parades in which the target was present were more likely to be accurate compared to those where the target was absent, there was negligible evidence supporting the hypothesis that a meaningful interaction effect existed between target presence and the sample duration conditions. Overall, these results show that there is no meaningful difference in binary accuracy between the sample duration conditions. Inferring from the interactions model, the most probable parameter values (for all conditions with corresponding 95\% HDIs are illustrated in Figure 1.

\section{Figure $1^{7}$}

Voice identification accuracy for 15s, 30s, and 60s sample duration conditions for target-present and-absent parades, Experiment 1 (nine-voice parade).

\footnotetext{
${ }^{6}$ Negligible here refers to a Bayes Factor in support of the alternative hypothesis $\left(\mathrm{BF}_{10}\right)$ being less than 3 ; while a $\mathrm{BF}_{01}$ greater than 3 can technically be interpreted as evidence in support of the null hypothesis, we argue that accepting the null hypothesis requires substantially more data points per cell.

${ }^{7}$ Note that we include the figures here for the benefit of the reviewers. The high-resolution images are uploaded separately.
} 


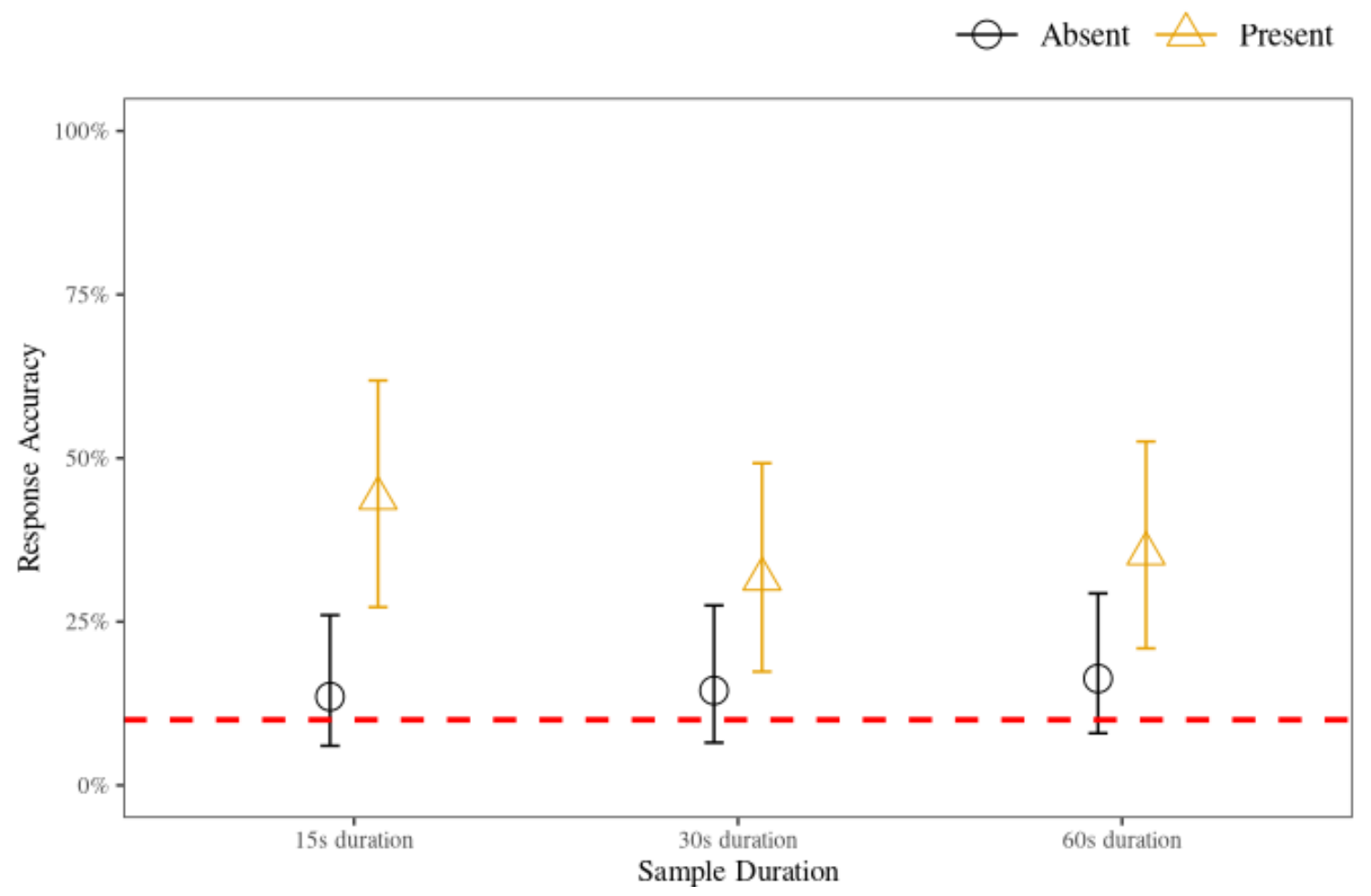

Note. The data points indicate the most likely posterior probability parameter values derived from the interaction model and the error bars show the 95\% Highest Density Interval (HDI). The dashed line indicates chance-level (10\%) accuracy rates ( $\left.1 / \mathrm{n}_{\text {response-options }}\right)$.

\section{Signal Detection Theory Model}

We evaluated voice-identification in the context of equal variance Gaussian signal detection theory (EVSDT, e.g., Wixted et al., 2016). Bayesian generalised mixed-effects models (with a Bernoulli distribution) were used to infer the model parameters. Model parameters were the response criterion $c$ (calculated as the negative standardised FA rate; DeCarlo, 1998), and $d$ ' (the difference between the standardised hit rate and the standardised FA rate) which represents listeners' ability to detect the signal (i.e., the target voice in target-present parades, or lack of a target voice in target absent parades) amongst noise (the foils). The model was implemented as non-linear syntax. The full decision frequency for all conditions is displayed in Table 1. 
For the SDT analyses, we removed target-present foil IDs so that the parameter of interest is purely the ability of listeners to discriminate between guilty suspects and innocent suspects (e.g., Colloff et al., 2016) and not an absolute notion of discriminability (i.e., the ability to discriminate between guilty suspects, innocent suspects, and foils). As we did not include a designated innocent suspect in the target absent parades, we applied a conventional nominal adjustment within the model syntax which adjusted the false alarm rate according to the number of voices in the parade.

\section{Table 1}

Decision frequency with percentages in parentheses (Experiment 1; nine-voice parades).

\begin{tabular}{|c|c|c|c|c|c|}
\hline \multirow[b]{2}{*}{ Sample Duration } & \multicolumn{3}{|c|}{ Target-Present } & \multicolumn{2}{|c|}{ Target-Absent } \\
\hline & Hit & Foil & Reject & Foil & Reject \\
\hline 15 seconds & $20(45 \%)$ & $21(48 \%)$ & $3(7 \%)$ & $42(87 \%)$ & $6(13 \%)$ \\
\hline 30 seconds & $14(32 \%)$ & $26(59 \%)$ & $4(9 \%)$ & $40(85 \%)$ & $7(15 \%)$ \\
\hline 60 seconds & $17(37 \%)$ & $27(59 \%)$ & $2(4 \%)$ & $35(83 \%)$ & $7(17 \%)$ \\
\hline Total & $51(38 \%)$ & $74(55 \%)$ & $9(7 \%)$ & $117(85 \%)$ & $20(15 \%)$ \\
\hline
\end{tabular}

Note. TP Hits $=$ correct target IDs, TP Foils $=$ incorrect Foil IDs, TP Reject $=$ incorrect rejection;

TA Foils $=$ incorrect foil IDs; TA Reject $=$ correct rejections.

The conditional maximum a posteriori ${ }^{8}$ estimates are presented in Table 2. For the parameter-value estimates we observe that there is strong evidence supporting the hypothesis that $d^{\prime}$ 'was above zero for the $15 \mathrm{~s}$ and 60 s duration conditions, but negligible evidence for the $30 \mathrm{~s}$

\footnotetext{
${ }^{8}$ For all inferences, we present the maximum a posteriori, which is a point estimate of an unknown quantity that is equivalent to the mode of the posterior distribution.
} 
condition. This indicates that, on average, listeners in the $15 \mathrm{~s}$ and $60 \mathrm{~s}$, but not the $30 \mathrm{~s}$ duration conditions, displayed some ability to distinguish the signal from the noise. As for criterion, there is moderate evidence supporting the hypothesis that the criterion for the 15 s duration is below zero, but negligible evidence for the 30 s and 60 s duration conditions. In other words, there is evidence that listeners in the $15 \mathrm{~s}$, but not the $30 \mathrm{~s}$ and 60 s conditions, adopted a liberal decision criterion. We found negligible differences when conducting pairwise comparisons for signal and criterion strength across sample durations $\left(\mathrm{BF}_{10}<3\right)$.

\section{Table 2}

Bayesian estimates of the EVSDT model analysis for Experiment 1 (nine-voice parades).

Criterion c represents willingness to respond target-present and d' indicates signal sensitivity.

\begin{tabular}{|c|c|c|c|c|c|c|c|c|}
\hline \multirow[b]{2}{*}{ Condition } & \multicolumn{4}{|c|}{ Sensitivity (d') } & \multicolumn{4}{|c|}{ Criterion (c) } \\
\hline & $\hat{\beta}$ & $95 \% \mathrm{HDI}$ & $\mathrm{BF}_{01}$ & $\mathrm{BF}_{10}$ & $\hat{\beta}$ & $95 \% \mathrm{HDI}$ & $\mathrm{BF}_{01}$ & $\mathrm{BF}_{10}$ \\
\hline 15 seconds & 1.15 & {$[0.40-2]$} & 0.04 & 23.6 & -0.13 & {$[-0.21--0.07]$} & 0.21 & 4.71 \\
\hline 30 seconds & 0.79 & {$[0.12-1.61]$} & 0.61 & 1.65 & -0.12 & {$[-0.20--0.06]$} & 0.55 & 1.81 \\
\hline 60 seconds & 1.28 & {$[0.42-2.25]$} & 0.08 & 12.17 & -0.11 & {$[-0.19--0.05]$} & 0.71 & 1.42 \\
\hline
\end{tabular}

Note. $\hat{\beta}=$ maximum a posteriori $(\mathrm{MAP}) ; 95 \% \mathrm{HDI}=95 \%$ Highest Density Interval; $\mathrm{BF}_{01}=$ Support for $\mathrm{H}_{0} ; \mathrm{BF}_{10}=$ Support for $\mathrm{H}_{1}$.

\section{Confidence}

Confidence ratings, on a scale of 0 (not at all confident) to 10 (extremely confident), were analysed in cumulative models for ordinal data (Bürkner \& Vuorre, 2019; Liddell \& Kruschke, 
2018). ${ }^{9}$ We investigated the relationship between confidence ratings and accuracy for each sample duration condition separately.

We found moderate evidence of a positive relationship between confidence and accuracy for the 60 s sample duration $(\hat{\beta}=.91$, HPDI: [0.15 -1.69$\left.], \mathrm{BF}_{10}=3.32\right)$. Evidence was negligible for the other sample durations (15s: $\hat{\beta}=.25$, HPDI: $[-0.48-0.96], \mathrm{BF}_{10}=0.23 ; 30 \mathrm{~s}: \hat{\beta}=.48$, HPDI: [-0.29 -1.25$\left.], \mathrm{BF}_{10}=0.41\right)$. In other words, participants were more confident about correct responses (than about incorrect responses) when listening to a voice parade with $60 \mathrm{~s}$ samples, but there was no meaningful relationship between confidence and accuracy for listeners in the 15s and 30s conditions. Posterior cell means are shown in Figure 2 for each condition.

Figure 2. Posterior confidence with 95\% HPDIs, Experiment 1 (nine-voice parade)

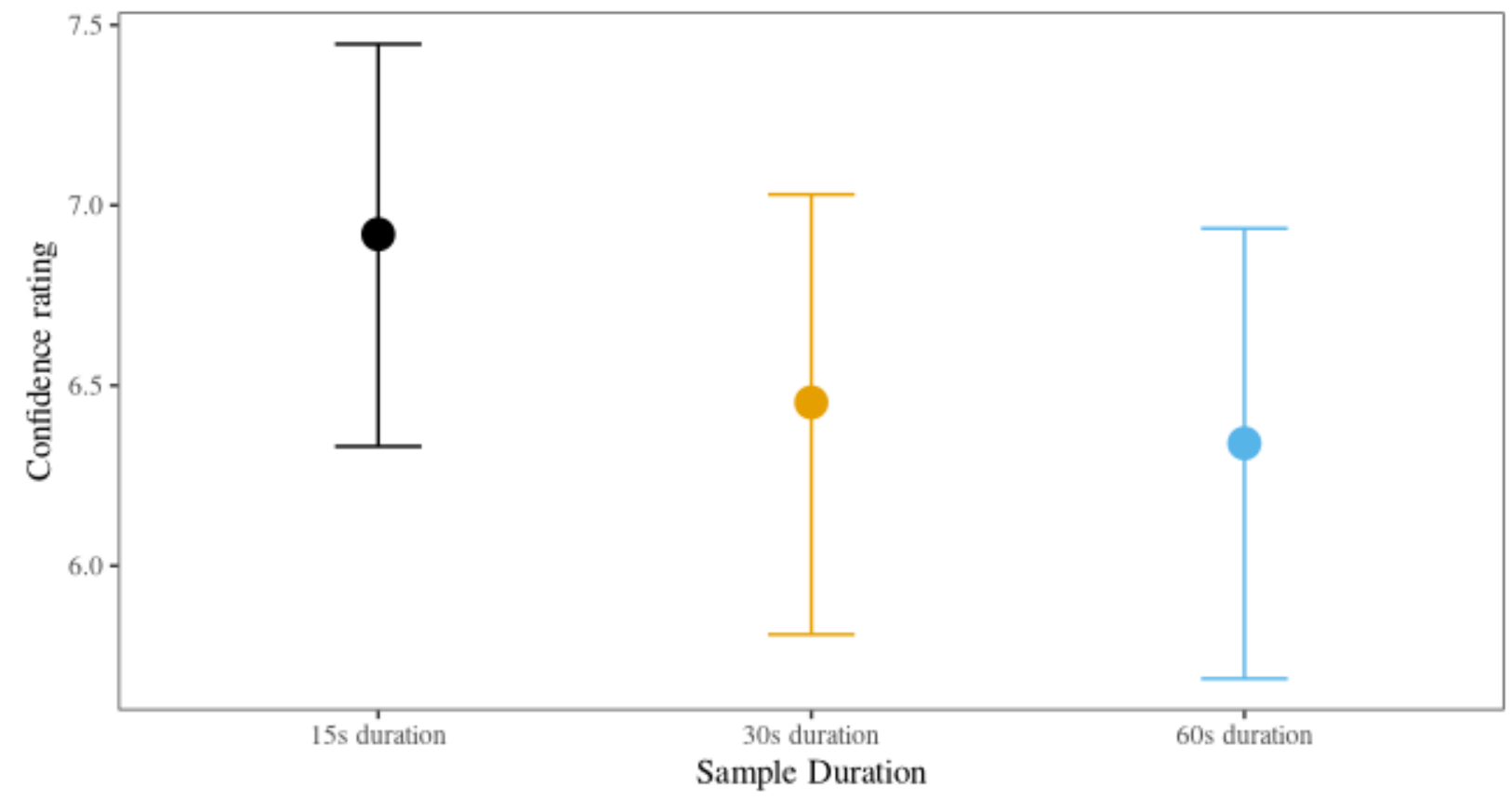

\footnotetext{
${ }^{9}$ While it is not uncommon for ordinal data to be analysed using methods that assume metric responses, this practice can lead to errors in inference (Liddel \& Kruschke, 2018).
} 


\section{Discussion}

The traditional binary analysis showed that accuracy rates were low $(<50 \%)$ and performance in parades where the target was absent was substantially lower than in parades where the target was present, consistent with previous findings (Kerstholt et al., 2004, 2004; Öhman et al., 2011, 2013a, 2013b; Perfect et al., 2002; Smith et al., 2020). Despite the overall low accuracy, all target-present parades had accuracy at above-chance levels. This indicates that even though the task used in the experiments was difficult - a short exposure to the target with incidental encoding, a cognitively demanding filler task, and quantitatively similar voices (i.e., a fair parade) - voice identification is difficult, but not impossible.

The signal detection analyses reveal that listeners in the $15 \mathrm{~s}$ and $60 \mathrm{~s}$ duration conditions were able to distinguish the 'guilty' voice from 'innocent' ones. However, in the 30s condition, listeners' ability to distinguish guilty from innocent was no different from chance. These findings partially support our hypothesis - based on temporal-ratio models of memory (Brown et al., 2007 ) - that the increased temporal distinctiveness of the shorter 15 s sample durations conditions offset the benefit of the maximal amount of identity information afforded by the longer 60s sample duration. Contrary to what one might expect, the results suggest that the 30 s sample duration condition does not represent the combined benefits of increased distinctiveness afforded by the shorter durations and increased identity information provided by the longer durations. Rather, the benefits of both the shorter and longer durations are reduced to a point where a compromise leads not to the 'best of both worlds' but to the worst.

We found that listeners in the $15 \mathrm{~s}$ sample duration condition were more likely to adopt a liberal response criterion compared to listeners in the 30s and 60s sample duration conditions, 
who had a neutral response criterion. This means that listeners in the $15 \mathrm{~s}$ sample duration condition had a heightened predisposition to respond that the target was present when undertaking a parade where the target was absent, translating to a higher likelihood of false alarming compared the other duration conditions. However, this should be treated as tentative due to the relatively small number of by-condition data points and considering the overlap of the density intervals between three sample duration conditions. As such, considering the similarity of listener discrimination between the shorter (15s) and longer (60s) durations, while at the same time taking into account the resource requirement of each, the benefits of the former appear to outweigh those of the latter.

There was a statistically meaningful relationship between confidence and accuracy in the 60s sample duration condition, but not in the $15 \mathrm{~s}$ or $30 \mathrm{~s}$ conditions. Listeners in the 60 s condition were more confident about accurate responses than inaccurate responses. The inconsistency of the results is consistent with the largely tenuous confidence-accuracy relationships reported in other earwitness research (Kerstholt et al. 2004; Ohman et al., 2011; Perfect et al., 2002). It is possible that longer exposure to the voice information in this sample condition, in conjunction with meaningful signal sensitivity, facilitated this confidence-accuracy relationship in the $60 \mathrm{~s}$ condition, but not the shorter sample duration conditions.

Overall, the results of this experiment provide initial evidence that shorter voice durations may be a feasible procedural change to the longer sample durations currently recommended in the Home Office (2003) guidelines. While further research is required to support this finding, we argue that the robust experimental design used in the present research, as well as the focus on creating ecologically valid voice parades using the expertise of forensic phonetic experts, is highly suggestive of a valid and reliable outcome. 


\section{Experiment 2}

In Experiment 2 we investigated if reducing the number of foils in a voice parade from eight to five would change the pattern of results found in Experiment 1. Here we hypothesised that due to the reduced number of voices, the auditory attentional demands (Zimmerman et al., 2016) would be reduced. This reduction in cognitive demand would result in fewer erroneous comparisons and subsequently facilitate a stronger signal sensitivity. In other words, we hypothesised that listeners undertaking a six-voice, as opposed to a nine-voice parade, would display stronger $d$ ' scores, as well as improved overall binary accuracy. However, based on the results of Experiment 1, we did not expect any by-duration differences to be found. The fact that we found no meaningful differences in binary accuracy between the six different parade groups suggests that while there may have been some inter-speaker variance, it was not statistically meaningful and unlikely to influence the findings above and beyond that accounted for as random effects in the various models. Additionally, we explored whether binary accuracy varied between the various target-groups which were treated as random effects in the various models. As the experimental design included target speakers in both early and late positions, and previous eyewitness research has found 'unwanted position effects' in sequential parades (Meisters et al., 2018) we also investigated if such position effects were present in the current data. Thus, following the primary analyses, we pooled the data from both experiments and collapsed the sample duration conditions to allow for a comparison between the two parade sizes, including looking at whether the position of the target affected witness performance, and whether this differed between nine- and six-voice parades. Finally, we explored whether binary accuracy varied between the various target-groups.

\section{Method}


Apart from the following exceptions, the materials and methods were identical to Experiment 1.

\section{Participants}

A total of $\mathrm{N}=278$ participants were recruited using Prolific.co. A total of eight participants had their data discarded (six for exceeding the between-voice response time manipulation check, and two due to non-completion of the filler task), making the final sample size $\mathrm{N}=270$. The average age of the participants was 28.82 years $(S D=6.27)$, with 134 males and 136 females.

\section{Procedure}

The parades contained six voices (one target, five foils in the target-present parade; six foils in the target-absent parade). To account for the reduced overall parade size, the target positions changed from positions three and seven in the nine-voice parade to positions two and five (Carlson et al., 2008; Smith, \& Baguley, 2014).

Whereas in Experiment 1 we constructed parades using the ten speakers assessed by listener participants as most similar-sounding to each other within each original group of 15 (MDS analysis; see Appendix A), in Experiment 2 we constructed parades using the seven speakers that listeners assessed as most similar-sounding to each other using the same set of similarity judgements, i.e. we effectively dropped from each parade the three speakers with the highest Euclidean distances from the target. The target speakers and replacement foils were the same in both experiments.

On the post-parade debriefing question (eliciting 1-5 Likert scale responses), descriptively speaking, participants found the target-absent parade (Mean=3.39, $S D=1.08)$ to be no more or less difficult than the target-present parade (Mean=3.38, $S D=1.02$ ). 


\section{Results}

\section{Accuracy}

We used the same analytic procedure here that was used in Experiment 1. Accurate parade identifications were scored as 1 and inaccurate identifications as 0 , in a 3 (sample duration: 15s, 30s, 60s) x 2 (target presence: present or absent) factorial design. This analysis treated the parade target (a total of six different parades and corresponding targets) as a random factor. Model predictors and their interaction terms were added incrementally to the interceptonly model and compared using cross-validation techniques. The fitted models' predictive performance was estimated as the sum of the expected log pointwise predictive density (elpd) alongside its standard error (SE). A model with a difference in $\mathrm{SE}(\Delta \mathrm{SE})$ equal to or greater than 5 is suggestive of better predictive performance (Vehtari et al., 2017). As in Experiment 1, while the model with only target presence included as a predictor resulted in the highest predictive performance, no model exceeded a $\triangle \mathrm{SE}$ of 5 (see Appendix $\mathrm{C}$, Table $\mathrm{C} 1$ for the full model comparison). For this reason, we selected the model which included the interaction effects.

The model, shown in full in Appendix C, Table C2, illustrate that there is negligible evidence to support the hypothesis that the 15 s or the 30 s sample durations differed in a statistically meaningful way from the reference category of 60s sample durations. While there was strong evidence to support the hypothesis that responses to parades in which the target was present were more likely to be accurate compared to those were the target was absent, there was negligible evidence supporting the hypothesis that a meaningful interaction effect existed between target presence and sample duration. Inferring from the interaction model, the most 
probable parameter values $(u)$ for all conditions with corresponding 95\% HDIs are illustrated in Figure 3.

\section{Figure 3}

Voice identification accuracy for 15s, 30s, and 60s sample duration conditions for target-present and-absent parades, Experiment 2 (six-voice parades).

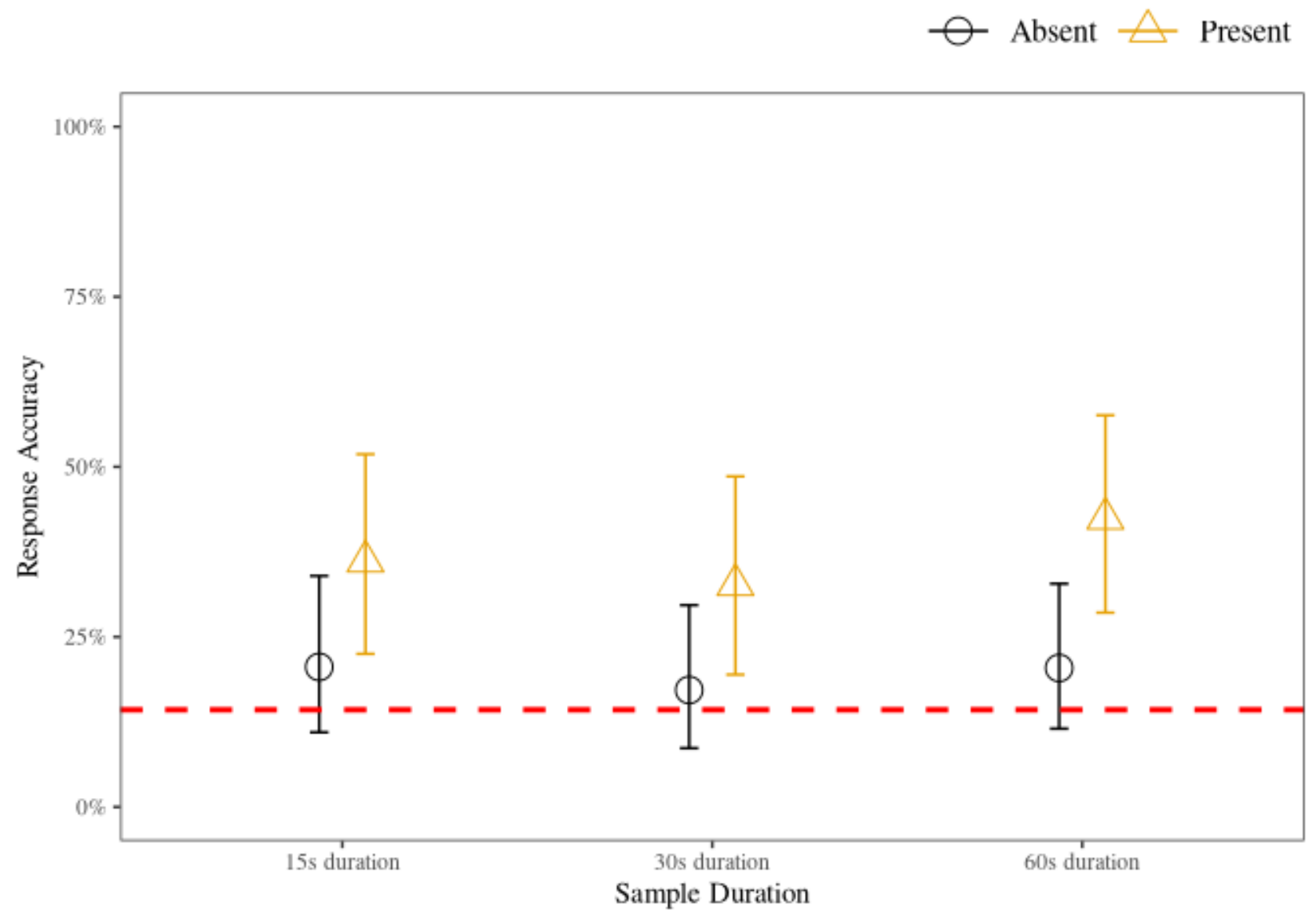

Note. The data points indicate the most likely posterior probability parameter values derived from the interaction model and the error bars show the 95\% Highest Density Interval (HDI). The dashed line indicates chance-level (14.2\%; $\left.1 / \mathrm{n}_{\text {response-options }}\right)$.

\section{Signal Detection Theory Model}


We used the same modelling approach as in Experiment 1. We evaluated voiceidentification in the context of equal variance Gaussian signal detection theory (EVSDT; e.g., Wixted et al., 2016). Model parameters were the response criterion $c$ and $d^{\prime}$. The model was implemented as non-linear syntax. The only difference was that the nominal parade size adjustment was for six voices and not nine voices. Again, we removed target-present foil IDs from the analysis to measure discrimination only between guilty and innocent suspects (e.g., Colloff et al., 2016). See Table 3 for the complete decision frequencies.

\section{Table 3}

Decision frequency with percentages in parentheses (Experiment 2, six-voice parades)

\begin{tabular}{lcccccc}
\hline & \multicolumn{2}{c}{ Target-Present } & & \multicolumn{2}{c}{ Target-Absent } \\
\cline { 2 - 4 } Sample Duration & Hit & Foil & Reject & & Foil & Reject \\
\hline 15 seconds & $16(36 \%)$ & $26(58 \%)$ & $3(7 \%)$ & & $36(78 \%)$ & $10(22 \%)$ \\
30 seconds & $14(33 \%)$ & $22(51 \%)$ & $7(16 \%)$ & & $37(82 \%)$ & $8(18 \%)$ \\
60 seconds & $21(46 \%)$ & $21(46 \%)$ & $4(9 \%)$ & & $37(82 \%)$ & $8(18 \%)$ \\
Total & $\mathbf{5 1 ( 3 8 \% )}$ & $\mathbf{6 9 ( 5 1 \% )}$ & $\mathbf{1 4}(\mathbf{1 0 \%})$ & $\mathbf{1 1 0}(\mathbf{8 1 \%})$ & $\mathbf{2 6}(\mathbf{1 9 \%})$ \\
\hline
\end{tabular}

The conditional maximum a posteriori estimates are presented in Table 4. As in Experiment 1, there was moderate to strong evidence in support of the hypothesis that signal sensitivity was significantly above zero in the $15 \mathrm{~s}$ and 60 s duration conditions, but negligible evidence that signal sensitivity differed from zero in the 30 s duration condition. We observed moderate evidence in support of the hypothesis that the response criterion is lower than zero for the 30s and 60s duration conditions, but negligible evidence for the $15 \mathrm{~s}$ duration condition. This 
suggests that, in contrast to Experiment 1, listeners in the 30 s and 60 s, but not the 15 s duration conditions, adopted a liberal decision criterion for all sample durations. Pairwise comparisons revealed no reliable differences for criterion or sensitivity $\left(\mathrm{BF}_{10}<3\right)$.

\section{Table 4}

Bayesian estimates of the EVSDT model analysis for Experiment 2 (six-voice parades). Criterion c represents willingness to respond target-present and d' indicates signal sensitivity.

\begin{tabular}{|c|c|c|c|c|c|c|c|c|}
\hline \multirow[b]{2}{*}{ Condition } & \multicolumn{4}{|c|}{ Sensitivity (d') } & \multicolumn{4}{|c|}{ Criterion (c) } \\
\hline & $\hat{\beta}$ & $95 \% \mathrm{HDI}$ & $\mathrm{BF}_{01}$ & $\mathrm{BF}_{10}$ & $\hat{\beta}$ & $95 \%$ HDI & $\mathrm{BF}_{01}$ & $\mathrm{BF}_{10}$ \\
\hline 15 seconds & 1.42 & {$[0.42,2.68]$} & 0.06 & 16.62 & -0.14 & {$[-0.22,-0.06]$} & 0.39 & 2.56 \\
\hline 30 seconds & 0.71 & {$[0.05,1.77]$} & 1.85 & 0.53 & -0.16 & {$[-0.24,-0.08]$} & 0.18 & 5.33 \\
\hline 60 seconds & 1.34 & {$[0.40,2.53]$} & 0.1 & 9.75 & -0.16 & {$[-0.24,-0.08]$} & 0.12 & 8.07 \\
\hline
\end{tabular}

Note. $\hat{\beta}=$ MAP; $95 \%$ HDI $=95 \%$ Highest Density Interval; $\mathrm{BF}_{01}=$ Support for $\mathrm{H}_{0} ; \mathrm{BF}_{10}=$ Support for $\mathrm{H}_{1}$.

\section{Confidence}

Confidence ratings, on a scale of 0 (not at all confident) to 10 (extremely confident), were analysed in cumulative models for ordinal data (Bürkner \& Vuorre, 2019; Liddell \& Kruschke, 2018).We investigated the relationship between confidence ratings and accuracy for each sample duration condition separately.

We found moderate evidence of a positive relationship between confidence and accuracy for the 15 s sample duration condition $\left(\hat{\beta}=.9\right.$, HPDI: $\left.[0.2-1.66], \mathrm{BF}_{10}=4.2\right)$. Evidence was 
negligible for all other sample durations (30s: $\hat{\beta}=.71$, HPDI: $[-0.05-1.5], \mathrm{BF}_{10}=0.23$;

60s: $\hat{\beta}=.48$, HPDI: [-0.54 -0.93$\left.], \mathrm{BF}_{10}=0.21\right)$. In other words, participants were more confident about correct responses (than about incorrect responses) when listening to a voice parade with 15s samples, but there was no meaningful relationship between confidence and accuracy for listeners in the 30s and 60s duration conditions. Posterior cell means are shown in Figure 4 for each condition.

Figure 4. Posterior confidence with 95\% HPDIs, Experiment 2 (six-voice parades)

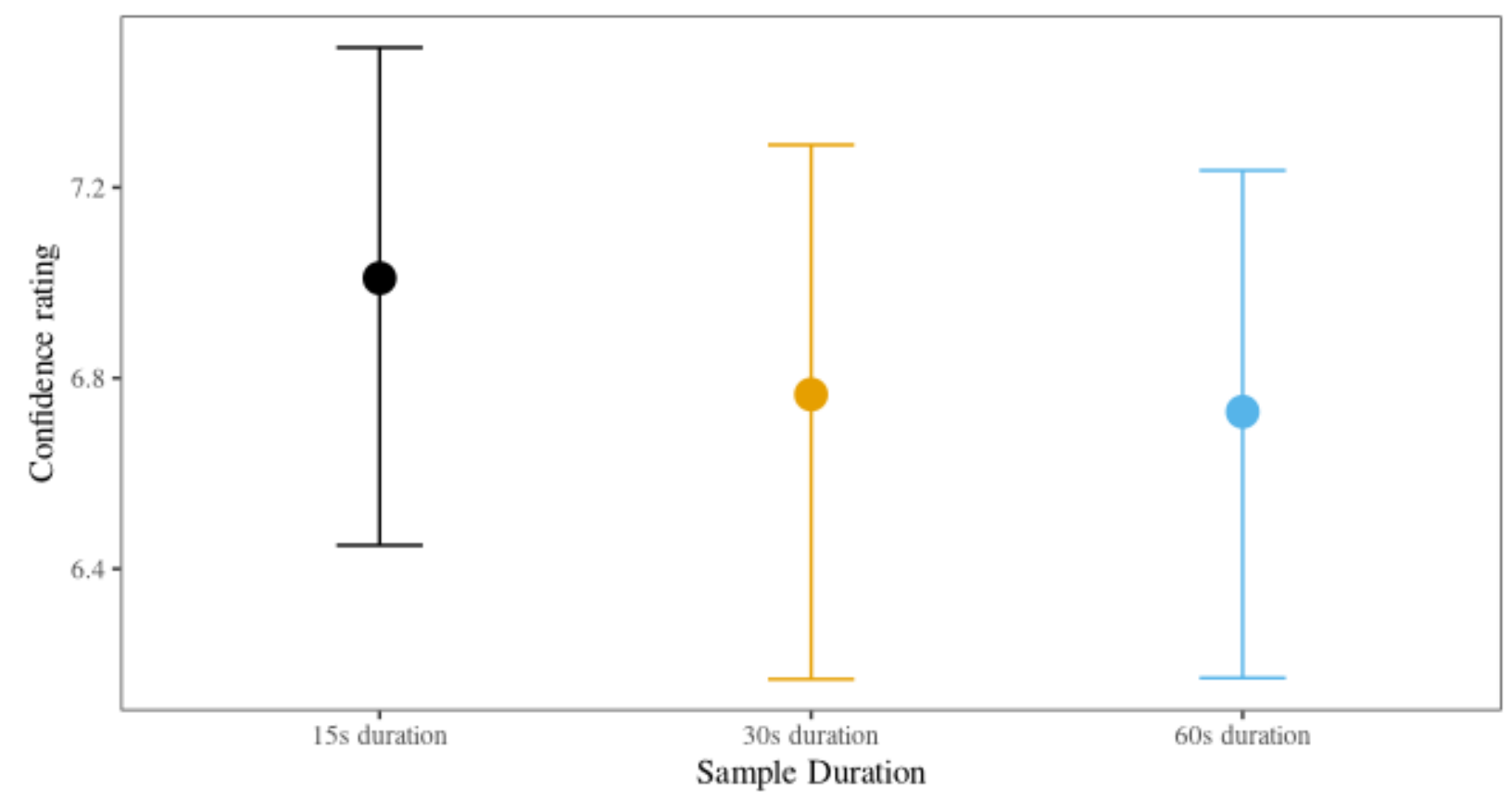

\section{Pooled Analysis}

As no meaningful differences in accuracy, sensitivity $\left(d^{\prime}\right)$, and criterion $(c)$ were found between the sample duration conditions in either experiment, we collapsed the data across parade size and investigated if any differences in sample duration would become evident with the larger sample size. Mirroring the analyses in Experiments 1 and 2, no meaningful differences in sample 
duration were found, nor were any interactions between target presence and the sample duration conditions; the only statistically meaningful predictor of accuracy was whether the parades contained a target or not. See Appendix D for the full results.

Next, we explored whether parade-group variation existed for binary accuracy. While there was variation in median accuracy between the different parade groups, there was no evidence to support the hypothesis that these differences were statistically meaningful (see Appendix D for the parameter value estimates and coinciding 95\% HDIs). See Appendix D, Figure D1, for an illustration of the cell means.

Finally, we investigated if positional effects played affected accuracy in target-present parades. Recall that both the nine- and six-voice parades had an 'early' voice position, voices three and two for the respective parade sizes, and a 'late' voice position, corresponding with voices seven and five respectively. We analysed these data using a similar approach to the accuracy analyses: we treated TP accuracy as a binary outcome $(0=$ accurate; $1=$ incorrect $)$, and included the predictors of position, parade size, and their interactions iteratively. The different targets were treated as a random factor. Leave-one-out cross validation was used to evaluate model comparisons (Vehtari et al., 2017). We found strong evidence supporting the hypothesis that parades which had targets in the later positions resulted in lower accuracy compared to parades which had targets in the earlier positions $\left(\hat{\beta}=-1.04,95 \% \mathrm{HDI}:-1.70--0.4, \mathrm{BF}_{10}=\right.$ 55.58). There was no evidence to suggest that accuracy differed between parade sizes $(\hat{\beta}=0.20$, 95\% HDI: $\left.-0.45-0.8, \mathrm{BF}_{10}=0.372, \mathrm{BF}_{01}=2.63\right)$, nor was there an interaction between target position and parade size $\left(\hat{\beta}=-0.39,95 \% \mathrm{HDI}:-1.39-0.42, \mathrm{BF}_{10}=0.79, \mathrm{BF}_{01}=1.26\right)$, suggesting that the positional effects were present and consistent in both parade sizes. These results are illustrated in Figure 5. 
Figure 5. Posterior accuracy with $95 \%$ HDIs between parade size conditions, combined data from Experiments 1 (nine voices) and 2 (six voices).

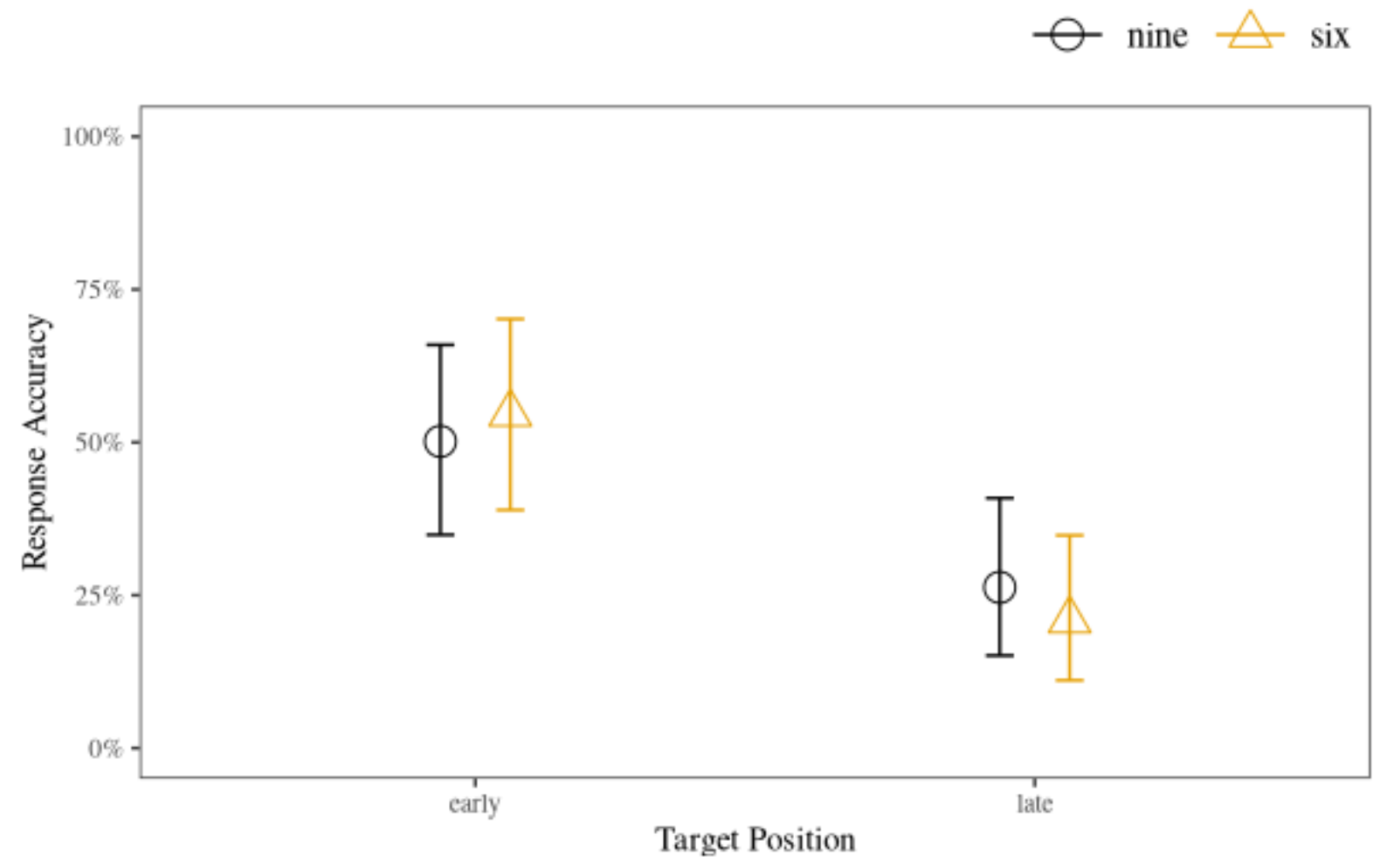

\section{Discussion}

As in the first experiment, the traditional binary analysis showed that accuracy rates were low $(<50 \%)$ and performance in parades where the target was absent was substantially lower than in parades where the target was present. All target-present parades, but no target-absent parades, had accuracy at above-chance levels. As expected, there were no by-duration differences in accuracy. Combining the data of both experiments, the pattern of outcomes was unchanged despite the increased sample size and subsequent precision of the parameter 
estimates. This suggests that regardless of whether the voices in the parade are $15 \mathrm{~s}, 30 \mathrm{~s}$, or $60 \mathrm{~s}$ in duration, there is unlikely to be a difference in accuracy across nine- or six-voice parades.

The pattern of sensitivity results found in the first experiment was replicated in the second experiment; that is, $d$ ' values were meaningfully above zero for the $15 \mathrm{~s}$ and $60 \mathrm{~s}$ conditions, but not for the 30 s condition. This reinforces the previous conclusion that the 30 s sample duration is not a suitable compromise between the shorter (15s) and longer (60s) duration samples. In partial alignment with our hypothesis, we found that the overall strength of signal sensitivity was higher for the $15 \mathrm{~s}$ and 60 s conditions in the six-voice parade compared to the nine-voice parade, while the 30 s condition had a reduced strength. However, the by-parade-size differences between sensitivity scores are unlikely to be statistically meaningful given the overlapping intervals. This suggests that the benefits of reduced auditory attentional demands (Zimmerman et al., 2016) provided by a parade with fewer voices are negligible.

We found that listeners in the 30 s and 60 s sample duration conditions were more likely to adopt a liberal response criterion. However, listeners in the $15 \mathrm{~s}$ sample duration condition displayed a neutral response criterion. This contrasts with Experiment 1 where only listeners in the $15 \mathrm{~s}$ duration condition displayed a liberal response criterion. Also standing in contrast with the nine-voice parade results, we found that listeners in the $15 \mathrm{~s}$ condition, but not the other sample durations, were more confident about accurate responses than inaccurate ones.

Importantly, we found strong evidence that 'unwanted position effects' are present were present in both experiments indicating that listeners in both the nine- and six-voice parades were substantially more likely to identify the target correctly when it appeared in an early position compared to a late position. While position effects have not been studied previously in voice 
identification research, there is some evidence that listeners are more likely to pick early faces in sequential eyewitness lineups (e.g., Nyman et al., 2020). We discuss the potential implications of these findings below.

\section{General Discussion}

We focused on the effect of two procedural manipulations on voice identification accuracy. Specifically, we tested how different sample durations (Experiment 1) and different parade sizes (Experiment 2) affect accuracy, signal detection measures, and self-rated confidence. Our motivation was to determine empirically whether the Home Office (2003) guidelines recommending 60 s voice samples and nine-voice parades could be amended to make voice parade construction less resource intensive, thereby reducing a potential delay - and subsequent memory decay - between initial exposure to a perpetrator's voice and subsequent voice identification procedure. We proposed that the reduced identity information afforded to listeners by shorter voice sample durations would be offset by the increased discriminability of each of the voices, as per temporal-ratio models of memory (Crowder, 2014; Bjork \& Whitten, 1974; Brown et al., 2007). We also proposed that having fewer voices in a parade would have a positive effect on identification performance due to 1) reduced attentional resources and 2) reduced risk of erroneous comparisons between the voice parade samples and the target voice (Zimmerman et al., 2016).

The results partially supported our hypotheses. In Experiment 1, we found that the performance on parades made up of 15-second samples were largely on par with performance on parades made up of 60-second samples, but signal sensitivity was at chance-levels for the 30second condition. As expected, the pattern of results found using a nine-voice parade in 
Experiment 1 was largely replicated using a six-voice parade Experiment 2, but, as hypothesized, sensitivity was marginally stronger for the 15 -second and 60 -second conditions. There were slight differences in criterion outcomes between the two experiments, but we argue that these were likely artefacts of the relatively small by-target-presence data points. Indeed, based on the underlying theoretical assumptions, the procedural manipulations applied in the two experiments would have had little, if any, effect on the criterion. We discuss the implications of these findings below.

The results of the binary accuracy analyses for both experiments underline the error prone nature of voice identification. Consistent with previous research on voice identification, our results reveal low overall accuracy and particularly high error-rates when the target is not present (e.g., Kerstholt et al., 2004, 2006; Ohman et al., 2011, 2013a, 2013b; Perfect et al., 2002; Smith et al., 2020). We found that, in terms of dichotomous accuracy, the shorter sample durations (i.e., 15s and 30s) are neither better nor worse than the Home Office recommended 60second duration. In both experiments, the results of the SDT analysis paint a slightly more nuanced picture, highlighting the value of moving away from a purely binary analytical approach. The signal detection analysis revealed that sensitivity - which in the present research represented the ability of listeners to discriminate between guilty suspects and innocent suspects (Colloff et al., 2016) - is significantly above chance in the shortest and longest, but not the middle-most sample durations. This suggests that there is a benefit of using shorter samples and a benefit of using longer samples, but these benefits seem to be reduced when attempting to reach a middle-ground 'sweet spot'. In terms of maximising listener identification performance, out of the three durations tested, the most effective sample duration to use in a voice parade would either be $15 \mathrm{~s}$ or $60 \mathrm{~s}$. 
The benefit of using the shorter samples, based on the temporal-ratio model of memory (Bjork and Whitten, 1974; Brown et al., 2007), is that these parade voices would be more distinct than voices played for a longer duration. This should allow for more effective relative and absolute judgements. In other words, identity information in shorter voice samples would be easier to compare against other voice samples in the parade as well as against the target voice heard at the beginning of the experiment (or the voice overheard in a real situation). The results appear support to this, but any benefit is limited only to the shortest voice samples and not the 'middle-ground' condition of 30s samples. The benefits of the longer sample durations would likely be the additional identity information available, allowing listeners to pick out variations of speech which may not be present in the shorter excerpts. From the evidence provided by the experiments, it appears that - assuming the temporal-ratio models of memory hold true - the hypothesized increase in distinctiveness provided by shorter samples may be as beneficial for the purposes of voice identification as the likely increased identity information afforded by the longer samples. There is likely to be a 'sweet spot' in terms of duration for both temporal distinctness and identity information, but the results of both Experiments 1 and 2 suggest that the 30s duration may miss both. If both short and long voice samples have similar identification performance outcomes, then it seems practical in terms of resource requirements for parade construction, as well as the subsequent facilitation of a shorter retention interval, that the shorter option should be advocated pending further empirical replications.

We also predicted that a parade with fewer voices would result in improved identification performance compared to a parade with a larger number of voices based on the idea that the parade fewer voices would free up cognitive demands relating to auditory attention (Zimmerman et al., 2016), allowing greater resources to be given to the fewer voices and reducing erroneous 
comparisons. While, as predicted, there were marginal increases in sensitivity strength for the $15 \mathrm{~s}$ and $60 \mathrm{~s}$ durations when presented in a six-voice parade, it is unlikely that these increases were statistically meaningful. We did not statistically compare these two outcomes as they were two separate experiments (and thus were subject to sampling bias), but the results appear to indicate that auditory attentional requirements to generate and compare identity-percepts from voices does not differ substantially between the two parade sizes.

A more fine-grained inspection of the accuracy rates between early and late positions in both six and nine-voice parades showed that earlier positions result in accuracy rates that were almost double the accuracy for later positions, indicating that serial voice parades suffer from the same 'unwanted position effects' found in sequential eyewitness lineups (Nyman et al., 2020; Meisters et al., 2018). One explanation for this is that more auditory attentional resources are available in the beginning of a voice parade compared to voices later in the parade. This increased attentional availability facilitates more robust identity-relevant information capture for these early positioned voices, while voices later in the parade receive less attention and subsequently weaker identity-percepts, possibly due to attentional fatigue and a corresponding decrease in task motivation (Moor et al, 2017). The fact that both the nine- and six-voice parades showed remarkably similar position effects would allow for this explanation, even if the attentional resource theory did not affect overall identification performance between the two parade sizes. Alternatively, using temporal models of memory as a base of interpretation, the decrease in accuracy for later positions may be due to the lack of a ratio-like mechanism (Bjork \& Whitten, 1974) being applied to the gaps between the voices. This 'flat gap' would result in the early positioned voices being substantially more distinct than voices in later positions. 
Regardless of the explanation, these positional effects are an important finding that warrant further inquiry.

The similar performance between six- and nine-voice parades might initially lead one to conclude that the six-voice parade should be recommended because it would reduce the amount of resources required to construct and administer the parade at no cost to identification performance. We argue, however, that because performance between the six- and nine-voice parades was so similar, the increased protection afforded to innocent suspects in the Home Office recommended nine-voice parade supersedes any benefits of reduced resource requirements provided by the smaller parade. To elaborate: if performance is at chance level, there is a greater likelihood of an innocent suspect being randomly selected in a six-voice parade (14.2\% chance of randomly selecting the innocent) compared to a nine-voice parade (10\% chance of randomly selecting the innocent). The fact that identification research in general has shown a robust 'proclivity to choose' effect (Baldassari et al., 2019) - evidenced in the present research by relatively consistent liberal criterion - and that identification when the target is not present is notoriously error-prone (Kerstholt et al., 2004, 2006; Öhman et al., 2011, 2013a, 2013b; Perfect et al., 2002; Smith et al., 2020) highlights the importance of safeguarding an innocent suspect from chance-selection.

It is important to highlight here that while target-absent accuracy was at chance level, target-present accuracy was consistently above chance across both experiments; that is, for all sample duration conditions and both parade sizes. The voice parades used in this experiment are very difficult, but still not impossible. Based on the related notions of propitious heterogeneity (Carlson et al., 2019) and filler siphoning (Wetmore et al., 2017), the similarity of the voices used in the present experiment is likely to make accurate identifications of a target voice 
particularly challenging even if the listener formed a robust memory of the voices. Indeed, the accuracy rates reported in the present study might be lower than those occurring in a 'real-world' situation. For instance, we would expect that the witness had been exposed to the perpetrator's voice for longer than one minute ${ }^{10}$ (as was the encoding duration used for both experiments) and it is feasible that the witness might have had some level of preparation to undertake the parade (i.e., rehearsing what they experienced) and subsequent intention to recall the voice heard at the crime scene (as opposed to current experiment being designed specifically to minimize rehearsal and reflection of the exposure). Additionally, the voices used as foils were drawn from a forensic database which matched age, gender, and location before being quantitatively assessed for similarity with the target voice. It seems unlikely that any 'real' voice parades would have such a homogenous selection of voice samples to choose from.

Confidence levels tended to fall within the middle of the scale used, possibly reflecting the uncertainty that listeners felt about their decision. The relationship between confidence and accuracy differed between the two experiments: in the nine-voice parade, there was a positive association between confidence and accuracy for the 60s sample duration, and in the six-voice parade there was a positive association for the $15 \mathrm{~s}$ sample duration. It seems unlikely that the size of size of the parade would have influenced the confidence-accuracy relationship between these different sample durations. More likely, the confidence-accuracy relationship is unreliable, possibly because listeners do not have the metacognitive awareness of just how difficult the voice parade is. Indeed, voice recognition research using unfamiliar voices often shows unreliable confidence-accuracy relationships, ranging from weak or null relationships (Smith et

\footnotetext{
${ }^{10}$ This is because forensic experts would generally not consider asking a witness to undertake a voice parade if they were only exposed to the perpetrator's voice for a brief period of time.
} 
al., 2020; Kerstholt et al., 2004; Öhman et al., 2011; Olsson et al., 1998) to significant relationships (Yarmey, 1991; Bull \& Clifford, 1984; Saslove \& Yarmey, 1980).

The current research illustrates the benefits that an interdisciplinary approach can have, particularly when concerns of ecologically valid parade construction and sample selection arise. We feel strongly that the research presented in this paper offers a procedural and methodological framework for undertaking evidence-based approaches to improving voice identification procedures. Undoubtedly, one of the major focal points of future voice identification parade research needs to be on improving the false alarm rate. The high choosing rates evidenced by the statistically meaningful liberal criterion value and a general inability to identify that the perpetrator's voice is not present at levels that are above-chance, compounded with the fact that target-absent parades are simulations of an innocent suspect scenario, paints a picture which highlights the urgent need for voice identification procedures to be updated.

Despite attempts to overcome many of the weaknesses prevalent in voice identification research, the experiments we present still have some limitations. However, we are confident that these limitations do not undermine the conclusions we draw. Firstly, the sample sizes for both experiments were determined by the general norms in the literature (i.e., what Lakens, 2021, calls heuristic justification) and the resource constraints (i.e., the cost). While the sample size used for both experiments was either on par with, or exceeded, those used in many previous voice identification experiments (e.g., Perfect et al., 2002; Kerstholt et al., 2006; Philippon et al., 2013; Smith et al., 2020), in some cases there was insufficient evidence to support either the null or alternative hypotheses. While future research should strive for sample sizes large enough to provide meaningful evidence one way or the other, we argue that any effect with sufficient strength and reliability to prompt a procedural change would have been observed with the current 
sample size. Secondly, while filler tasks are commonly used to mimic the effects of a 'real' temporal retention interval (e.g., Smith et al., 2020) and reduce experimental costs, it is important that any findings which are used to influence policy changes are first replicated using more ecologically valid intervals extending to days or even weeks. That said, the five-minute filler task used in the present research is not uncommon (e.g., Smith et al., 2020; Philippon et al., 2013; McAllister, 1993) and is sufficient for the memory of the 'perpetrator's voice' heard in the beginning of the experiment to be stored in long-term memory (the duration of short-term memory has been estimated to be about 10-30s; Jonides et al., 2008).

\section{Conclusion}

The current paper adds to the slowly growing literature which highlights the value of system variable research in voice identification. The experiments undertaken were novel as they directly compared United Kingdom Home Office guidelines for constructing voice parades against less resource-demanding alternatives. Importantly, the development of the parades and the selection and editing of the voice samples, from a psychological perspective, is novel due to the strong interdisciplinary approach that was adopted and implemented in all stages. Through this collaboration, we have outlined a method of parade construction and voice selection that is both rigorous, replicable, and maximises ecologically validity. We provide initial evidence indicating that the voice sample duration recommended by the Home Office to be used in voice parades may be reduced without decreasing successful identification rates, while at the same time potentially saving time and other police resources. We also demonstrate that there is currently no empirical justification to support the idea that parade size be reduced from the recommended nine voices, at least until further research identifies how target-absent false alarm rates can be substantially and reliably reduced. 


\section{Data Availability Statement}

The data and $\mathbf{R}$ scripts that support the findings of this study are openly available at https://osf.io/vh8ge/?view only=3b33eb68a0404f82be1f1ecb26d8c6e9 [This will be unblinded after peer review]. 


\section{References}

Anwyl-Irvine, A. L., Massonnié, J., Flitton, A., Kirkham, N., \& Evershed, J. K. (2020). Gorilla in our midst: An online behavioral experiment builder. Behavior Research Methods, 52(1), 388-407. https://doi.org/10.3758/s13428-019-01237-X

Baldassari, M. J., Kantner, J., \& Lindsay, D. S. (2019). The importance of decision bias for predicting eyewitness lineup choices: toward a Lineup Skills Test. Cognitive Research: Principles and Implications, 4(1), 1-13.

Bestelmeyer, P. E. G., Rouger, J., DeBruine, L. M., \& Belin, P. (2010). Auditory adaptation in vocal affect perception. Cognition, 117(2), 217-223.

https://doi.org/10.1016/j.cognition.2010.08.008

Bjork, R. A., \& Whitten, W. B. (1974). Recency-sensitive retrieval processes in long-term free recall. Cognitive Psychology, 6(2), 173-189. https://doi.org/10.1016/00100285(74)90009-7

Broeders, A., \& van Amelsvoort, A. (2001). A practical approach to forensic earwitness identification: Constructing a voice line-up. Problems of Forensic Sciences, 47, 237-245.

Brown, G. D. A., Neath, I., \& Chater, N. (2007). A temporal ratio model of memory. Psychological Review, 38. https://doi.org/10.1037/0033-295X.114.3.539

Bull, R., \& Clifford, B. R. (1984). Earwitness voice recognition accuracy. Eyewitness Testimony: Psychological Perspectives, 92-123. 
Bürkner, P.-C., \& Vuorre, M. (2019). Ordinal regression models in Psychology: A tutorial. Advances in Methods and Practices in Psychological Science, 25. http://dx.doi.org/10.1177/2515245918823199

Calderwood, L., McKay, D. R., \& Stevenage, S. V. (2019). Children's identification of unfamiliar voices on both target-present and target-absent lineups. Psychology, Crime \& Law, 25(9), 896-910. https://doi.org/10.1080/1068316X.2019.1597090

Cantone, J. A. (2010). Do you hear what I hear: Empirical research on earwitness testimony. Tex. Wesleyan L. Rev., 17, 123.

Carlson, C. A., Gronlund, S. D., \& Clark, S. E. (2008). Lineup composition, suspect position, and the sequential lineup advantage. Journal of Experimental Psychology: Applied, 14(2), 118.

Carlson, C. A., Jones, A. R., Whittington, J. E., Lockamyeir, R. F., Carlson, M. A., \& Wooten, A. R. (2019). Lineup fairness: propitious heterogeneity and the diagnostic featuredetection hypothesis. Cognitive research: principles and implications, 4(1), 20. https://doi.org/10.1186/s41235-019-0172-5

Cohen, A. L., Starns, J. J., Rotello, C. M., \& Cataldo, A. M. (2020). Estimating the proportion of guilty suspects and posterior probability of guilt in lineups using signal-detection models. Cognitive Research: Principles and Implications, 5(1), 1-24. https://doi.org/10.1186/s41235-020-00219-4

Colloff, M. F., Wade, K. A., Strange, D. (2016). Unfair lineups make witnesses more likely to confuse innocent and guilty suspects. Psychological Science, 27, 1227-1239. doi:10.1177/0956797616655789

Cook, S., \& Wilding, J. (1997a). Earwitness Testimony 2: Voices, Faces and Context. 17. 
Cook, S., \& Wilding, J. (1997b). Earwitness testimony: Never mind the variety, hear the length. Applied Cognitive Psychology: The Official Journal of the Society for Applied Research in Memory and Cognition, 11(2), 95-111.

Crowder, R. G. (2014). Principles of Learning and Memory. doi:10.4324/9781315746944

DeCarlo, L. T. (1998). Signal detection theory and generalized linear models. Psychological Methods, 3(2), 186-205. https://doi.org/10.1037/1082-989X.3.2.186

Gelman, A., Hwang, J., \& Vehtari, A. (2014). Understanding predictive information criteria for Bayesian models. Statistics and Computing, 24(6), 997-1016. https://doi.org/10.1007/s11222-013-9416-2

Gold, E., Ross, S., \& Earnshaw, K. (2018). The 'West Yorkshire Regional English Database': Investigations into the generalizability of reference populations for forensic speaker comparison casework. Interspeech 2018: Speech Research for Emerging Markets in Multilingual Societies, 2748-2752.

Hammersley, R., \& Read, J. D. (1996). Voice identification by humans and computers. In S. L. Sporer, R. S. Malpass, \& G. Koehnken (Eds.), Psychological issues in eyewitness identification (pp. 117-152). Lawrence Erlbaum Associates, Inc.

Hoffman, H. J., Dobie, R. A., Losonczy, K. G., Themann, C. L., \& Flamme, G. A. (2017). Declining prevalence of hearing loss in US adults aged 20 to 69 years. JAMA Otolaryngology Head and Neck Surgery, 143(3), 274-285. doi:10.1001/jamaoto.2016.3527

Home Office. (2003). Home Office circular 057/2003: Advice on the use of voice identification parades. 
http://webarchive.nationalarchives.gov.uk/20130308000037/http://www.homeoffice.gov. uk/about-us/corporate-publications-strategy/home-office-circulars/circulars-2003/0572003/

Kelly, S. (2018). Investigating the phonetic and linguistic features used by speakers to communicate an intent to harm. Ph.D. Dissertation, University of York. https://etheses.whiterose.ac.uk/23920/?msclkid=10e0c388c21811ec9f11a6c3ef5117b5

Kerstholt, J. H., Jansen, N. J., Van Amelsvoort, A. G., \& Broeders, A. P. A. (2004). Earwitnesses: Effects of speech duration, retention interval and acoustic environment. Applied Cognitive Psychology: The Official Journal of the Society for Applied Research in Memory and Cognition, 18(3), 327-336.

Kerstholt, J. H., Jansen, N. J., Van Amelsvoort, A. G., \& Broeders, A. P. A. (2006). Earwitnesses: Effects of accent, retention and telephone. Applied Cognitive Psychology: The Official Journal of the Society for Applied Research in Memory and Cognition, 20(2), 187-197.

King, D. L., Jones, F. L., Pearlman, R. C., Tishman, A., \& Felix, C. A. (2002). The length of the retention interval, forgetting, and subjective similarity. Journal of Experimental Psychology: Learning, Memory, and Cognition, 28(4), 660-671.

https://doi.org/10.1037/0278-7393.28.4.660

Lakens, D. (2021). Sample Size Justification [Preprint]. PsyArXiv. https://doi.org/10.31234/osf.io/9d3yf

Laub, C. E., Wylie, L. E., \& Bornstein, B. H. (2013). Can the courts tell an ear from an eye: Legal approaches to voice identification evidence. Law \& Psychol. Rev., 37, 119. 
Liddell, T. M., \& Kruschke, J. K. (2018). Analyzing ordinal data with metric models: What could possibly go wrong? Journal of Experimental Social Psychology, 79, 328-348. https://doi.org/10.1016/j.jesp.2018.08.009

Macmillan, N.A., \& Creelman, C.D. (2004). Detection Theory: A User's Guide (2nd ed.). Psychology Press. https://doi.org/10.4324/9781410611147

Makowski, D., Ben-Shachar, M. S., Chen, S. H., \& Lüdecke, D. (2019). Indices of effect existence and significance in the Bayesian framework. Frontiers in psychology, 2767. https://doi.org/10.3389/fpsyg.2019.02767

McAleer, P., Todorov, A., \& Belin, P. (2014). How Do You Say 'Hello’? Personality Impressions from Brief Novel Voices. PLOS ONE, 9(3), 9.

McAllister, H. A., Dale, R. H., \& Keay, C. E. (1993). Effects of lineup modality on witness credibility. The Journal of Social Psychology, 133(3), 365-376.

Mansour, J. K., Beaudry, J. L., \& Lindsay, R. C. L. (2017). Are multiple-trial experiments appropriate for eyewitness identification studies? Accuracy, choosing, and confidence across trials. Behavior Research Methods, 49(6), 2235-2254. https://doi.org/10.3758/s13428-017-0855-0

McDougall, K. (forthcoming) 'Ear-catching versus eye-catching? Some developments and current challenges in earwitness identification evidence' To appear in Proceedings of XVII AISV (Associazione Italiana Scienze della Voce) Conference: 'Speaker Individuality in Phonetics and Speech Sciences: Speech Technology and Forensic Applications', 4-5 February 2021, University of Zürich. 
McDougall, K. (2013) ‘Assessing perceived voice similarity using multidimensional scaling for the construction of voice parades.' International Journal of Speech, Language and the Law 20.2: 163-172.

McDougall, K., Nolan, F., \& Hudson, T. (2015). Telephone transmission and earwitnesses: performance on voice parades controlled for voice similarity. Phonetica, 72(4), 257-272. https://doi.org/10.1159/000439385

McElreath, R. (2016). Statistical rethinking: A Bayesian course with examples in R and Stan. CRC Press/Taylor \& Francis Group.

McGorrery, P. G., \& McMahon, M. (2017). A fair 'hearing' Earwitness identifications and voice identification parades. The International Journal of Evidence \& Proof, 21(3), 262-286.

Meissner, C. A., Tredoux, C. G., Parker, J. F., \& MacLin, O. H. (2005). Eyewitness decisions in simultaneous and sequential lineups: A dual-process signal detection theory analysis. Memory \& Cognition, 33(5), 783-792.

Meisters, J., Diedenhofen, B., \& Musch, J. (2018). Eyewitness identification in simultaneous and sequential lineups: an investigation of position effects using receiver operating characteristics. Memory (Hove, England), 26(9), 1297-1309. https://doi.org/10.1080/09658211.2018.1464581

Memon, A., \& Yarmey, A. D. (1999). Earwitness recall and identification: Comparison of the cognitive interview and the structured interview. Perceptual and Motor Skills, 88(3), 797-807.

Moore, T. M., Key, A. P., Thelen, A., \& Hornsby, B. (2017). Neural mechanisms of mental fatigue elicited by sustained auditory processing. Neuropsychologia, 106, 371-382. https://doi.org/10.1016/j.neuropsychologia.2017.10.025 
Nolan, F. (2003). A recent voice parade. The International Journal of Speech, Language and the Law, 10(2), 277-291. https://doi.org/10.1558/s1l.2003.10.2.277

Nolan, F., McDougall, K., de Jong, G., \& Hudson, T. (2009). The DyViS database: Stylecontrolled recordings of 100 homogeneous speakers for forensic phonetic research. The International Journal of Speech, Language and the Law, 16(1), 31-57. https://doi.org/10.1558/ijsll.v16i1.31

Nolan, F., McDougall, K., \& Hudson, T. (2013). Effects of the telephone on perceived voice similarity: Implications for voice line-ups. The International Journal of Speech, Language and the Law, 20(2), 229-246. https://doi.org/10.1558/ijsll.v20i2.229

Nyman, T. J., Antfolk, J., Lampinen, J. M., Korkman, J., \& Santtila, P. (2020). Line-up image position in simultaneous and sequential line-ups: the effects of age and viewing distance on selection patterns. Frontiers in psychology, 1349. https://doi.org/10.3389/fpsyg.2020.01349

Öhman, L., Eriksson, A., \& Granhag, P. A. (2011). Overhearing the planning of a crime: do adults outperform children as earwitnesses? Journal of Police and Criminal Psychology, 26(2), 118-127. https://doi.org/10.1007/s11896-010-9076-5

Öhman, L., Eriksson, A., \& Granhag, P. A. (2013a). Angry voices from the past and present: Effects on adults' and children's earwitness memory. Journal of Investigative Psychology and Offender Profiling, 10(1), 57-70.

Öhman, L., Eriksson, A., \& Granhag, P. A. (2013b). Enhancing Adults' and Children's Earwitness Memory: Examining Three Types of Interviews. Psychiatry, Psychology and Law, 20(2), 216-229. https://doi.org/10.1080/13218719.2012.658205 
Olsson, N. (2000). A comparison of correlation, calibration, and diagnosticity as measures of the confidence-accuracy relationship in witness identification. Journal of Applied Psychology, 85(4), 504. https://doi.org/10.1037/0021-9010.85.4.504

Olsson, N., Juslin, P., \& Winman, A. (1998). Realism of Confidence in Earwitness Versus Eyewitness Identification. Journal of Experimental Psychology: Applied, 4(2), 18.

Perfect, T. J., Hunt, L. J., \& Harris, C. M. (2002). Verbal overshadowing in voice recognition. Applied Cognitive Psychology, 16(8), 973-980. https://doi.org/10.1002/acp.920

Philippon, A. C., Cherryman, J., Bull, R., \& Vrij, A. (2007). Earwitness identification performance: The effect of language, target, deliberate strategies and indirect measures. Applied Cognitive Psychology, 21(4), 539-550. https://doi.org/10.1002/acp.1296

Philippon, A. C., Randall, L. M., \& Cherryman, J. (2013). The Impact of Laughter in Earwitness Identification Performance. Psychiatry, Psychology and Law, 20(6), 887-898. https://doi.org/10.1080/13218719.2013.768194

Robson, J. (2016). A Fair Hearing? The Use of Voice Identification Parades in Criminal Investigations in England and Wales. 1, 15.

Saslove, H., \& Yarmey, A. D. (1980). Long-term auditory memory: speaker identification. Journal of Applied Psychology, 65(1), 111. https://doi.org/10.1037/0021-9010.65.1.111

Sherrin, C. (2015). Earwitness evidence: the reliability of voice identifications. SSRN Electronic Journal. https://doi.org/10.2139/ssrn.2628313

Skuk, V. G., \& Schweinberger, S. R. (2013). Adaptation aftereffects in vocal emotion perception elicited by expressive faces and voices. PLOS ONE, 8(11), e81691. https://doi.org/10.1371/journal.pone.0081691 
Smith, H. M. J., \& Baguley, T. (2014). Unfamiliar voice identification: Effect of post-event information on accuracy and voice ratings. Journal of European Psychology Students, 5(1), 59-68. https://doi.org/10.5334/jeps.bs

Smith, H. M. J., Bird, K., Roeser, J., Robson, J., Braber, N., Wright, D., \& Stacey, P. C. (2020). Voice parade procedures: Optimising witness performance. Memory, 28(1), 2-17. https://doi.org/10.1080/09658211.2019.1673427

Sørensen, M. H. (2012). Voice line-ups: speakers' F0 values influence the reliability of voice recognitions. International Journal of Speech, Language \& the Law, 19(2). doi: 10.1558/ijsll.v19i2.145

Stevenage, S. V. (2018). Drawing a distinction between familiar and unfamiliar voice processing: A review of neuropsychological, clinical and empirical findings. Neuropsychologia, 116, 162-178. https://doi.org/10.1016/j.neuropsychologia.2017.07.005

Stevenage, S. V., Howland, A., \& Tippelt, A. (2011). Interference in eyewitness and earwitness recognition. Applied Cognitive Psychology, 25(1), 112-118.

Stevenage, S. V., Hugill, A. R., \& Lewis, H. G. (2012). Integrating voice recognition into models of person perception. Journal of Cognitive Psychology, 24(4), 409-419. https://doi.org/10.1080/20445911.2011.642859

Tunnicliff, J. L., \& Clark, S. E. (2000). Selecting foils for identification lineups: Matching suspects or descriptions? Law and Human Behavior, 24(2), 231-258.

https://doi.org/10.1023/A:1005463020252 
Van Wallendael, L. R., Surace, A., Parsons, D. H., \& Brown, M. (1994). 'Earwitness’ voice recognition: Factors affecting accuracy and impact on jurors. Applied Cognitive Psychology, 8(7), 661-677.

Vehtari, A., Gelman, A., \& Gabry, J. (2017). Practical Bayesian model evaluation using leaveone-out cross-validation and WAIC. Statistics and Computing, 27(5), 1413-1432. https://doi.org/10.1007/s11222-016-9696-4

Wagenmakers, E.-J., Lodewyckx, T., Kuriyal, H., \& Grasman, R. (2010). Bayesian hypothesis testing for psychologists: A tutorial on the Savage-Dickey method. Cognitive Psychology, 32.

Wells, G. L. (1978). Applied eyewitness-testimony research: System variables and estimator variables. Journal of Personality and Social Psychology, 36(12), 1546. https://doi.org/10.1037/0022-3514.36.12.1546

Wells, G. L. (1993). What do we know about eyewitness identification? American Psychologist, $48(5), 553$.

Wells, G. L., \& Windschitl, P. D. (1999). Stimulus sampling and social psychological experimentation. Personality and Social Psychology Bulletin, 25(9), 1115-1125. https://doi.org/10.1177/01461672992512005

Wetmore, S. A., McAdoo, R. M., Gronlund, S. D., \& Neuschatz, J. S. (2017). The impact of fillers on lineup performance. Cognitive research: principles and implications, 2(1), 113. https://doi.org/10.1186/s41235-017-0084-1

Wixted, J. T., Mickes, L., Dunn, J. C., Clark, S. E., \& Wells, W. (2016). Estimating the reliability of eyewitness identifications from police lineups. Proceedings of the National Academy of Sciences, 113(2), 304-309. https://doi.org/10.1073/pnas.1516814112 
Woods, K. J. P., Siegel, M. H., Traer, J., \& McDermott, J. H. (2017). Headphone screening to facilitate web-based auditory experiments. Attention, Perception, \& Psychophysics, 79(7), 2064-2072. https://doi.org/10.3758/s13414-017-1361-2

Yarmey, Daniel. (1991). Descriptions of distinctive and non-distinctive voices over time. Journal of the Forensic Science Society, 31(4), 421-428. https://doi.org/10.1016/S0015$\underline{7368(91) 73183-6}$

Zetterholm, E., Sarwar, F., Thorvaldsson, V., \& Allwood, C. M. (2012). Earwitnesses: the effect of type of vocal differences on correct identification and confidence accuracy.

International Journal of Speech, Language \& the Law, 19(2). doi: 10.1558/ijsll.v19i2.219

Zimmermann, J. F., Moscovitch, M., \& Alain, C. (2016). Attending to auditory memory. Brain Research, 1640, 208-221. https://doi.org/10.1016/j.brainres.2015.11.032 


\section{Appendix A}

\section{Multidimensional scaling analysis for speaker selection}

Three groups of 15 speakers were randomly selected from the DyViS database (DyViS groups1, 2 and 3), one group of 15 speakers was randomly selected from the YorViS database, and one group of 15 speakers from Bradford (WYRED 1) and another group of 15 speakers from Wakefield (WYRED 2) were randomly selected from the WYRED database. Except for YorViS where the database was too small to allow this, any particularly unusual-sounding speakers were omitted from the selection and further speakers selected randomly until each group contained 15 speakers. For each speaker, two short audio clips (labelled 'utterance 1 (U1)' and 'utterance 2 (U2)') of approximately three seconds were extracted from the mock interview studio-quality recordings. The subjects of all U1 and U2 samples were consistent across speakers (all U1 samples related to the speaker denying knowledge of a man named Robert Freeman; U2 speech involved the speaker denying having been at the Yewtree Reservoir on Wednesday evening). Within each 15-speaker group, each speaker was matched with all other speakers and with himself to form 120 pairings. Each pairing of speakers was represented by a U1 and a U2 (by random assignment). The order in which the two utterances were presented (U1, U2 or U2, U1) was determined by random assignment and allocated to one of two order blocks. Each listener participant had an equal chance of listening to either order block. The sequence list for each set was generated in random order on each occasion.

Participants were exposed to all possible voice utterances in random order during a 'practice' trial. An optional break was offered every 40-trials. The process was completed for the first DyViS group and the YorViS group in prior experiments in a lab environment (Nolan et al., 
used to present the stimuli to listeners for an additional two DyViS and two WYRED groups online. Online data collection was necessary due to the Covid-19 pandemic.

The online and lab-based procedures were largely the same, however, to ensure that participants who undertook the task online were using headphones and not loudspeakers, a headphone screening task (Woods et al., 2017) was implemented. In this task, participants were required to identify the softest tone correctly out of three options, at least four out of six times; it was not possible to identify the softest tone correctly using speakers. If a participant failed the headphone screener initially, they were allowed one additional attempt before being rejected from the experiment. Additionally, a progress bar was included in the online version of the task so that participants had an idea of the time left to complete the task.

The experiment was conducted one listener at a time in a quiet room for the lab-based data collection and the online participants were required to be in a quiet environment - the latter requirement was partially enforced via the headphone screener; the stimuli were played via headphones or earphones at a comfortable but clearly audible volume. Before beginning the online experiment, participants were asked to play a sample of static noise and calibrate their hardware volume so that it was clear, but comfortable.

Each listener was required to read a set of instructions explaining that they would be asked to compare a number of voices and assess their degree of similarity. Participants were required to listen to both voice pairs before making a similarity judgement. When the first voice utterance was played, a text appeared on the screening alerting the participant that this was 'Voice 1' and after a one-second gap, the second voice utterance played with a corresponding 'Voice 2' text message. After both utterances had been played, the next screen displayed the 
question 'How similar are these voices?' and, below, the numbers 1 (very similar) to 9 (very different). The listener was asked to mouse-click on a number to register their judgment and to move on to the next pair of voices. Before each pairing there was a silence of 1.5 seconds. A total of $\mathrm{N}=20$ participants (age range: $17-42$ years, approximately balanced for gender, speakers of English as a first language, based in Great Britain for most of their lives, no known speech or hearing difficulties) rated the utterances for each of the six sample groups.

After the similarity ratings had been collected, analyses using Multidimensional Scaling (MDS) were undertaken in SPSS to determine which ten out of each of the groups of 15 speakers were rated to be the most similar to each other (as per McDougall, 2013 and McDougall et al, 2015). For each group of 15 speakers, the speaker selected as the target was the one who yielded the shorter sum of Euclidean distances between himself and the nine speakers closest to him in the five-dimensional space generated by the MDS for that 15 -speaker group. The five speakers farthest from the target were discarded. The fifth (middlemost) speaker out of the nine most similarly rated speakers was selected as the replacement foil. Thus, each original 15-speaker group gave a set of ten speakers for use in an experimental parade: the speaker with whom all other voices were compared was the target voice, and all other speakers were foils except for the middlemost speaker, who was used as the replacement foil used in target-absent parades (see Tables A2 and A3 for the complete speaker list).

\section{Table A1}

An overview of the sample and recording characteristics of the forensic databases.

\begin{tabular}{lllll}
\hline Database Age Accent & $\begin{array}{l}\text { Number of } \\
\text { speakers }\end{array}$ & $\begin{array}{l}\text { Tasks relevant to the } \\
\text { present study }\end{array}$ & $\begin{array}{l}\text { Quality and Recording } \\
\text { Equipment }\end{array}$ \\
\hline
\end{tabular}




\begin{tabular}{|c|c|c|c|c|c|}
\hline DyViS & $\begin{array}{l}18- \\
25\end{array}$ & $\begin{array}{l}\text { Standard } \\
\text { Southern } \\
\text { British } \\
\text { English } \\
\text { (SSBE) }\end{array}$ & 100 & $\begin{array}{l}\text { Included: 1) Simulated } \\
\text { police interview, 2) } \\
\text { telephone call with } \\
\text { accomplice }\end{array}$ & $\begin{array}{l}\text { Studio quality (.wav, } 44.1 \mathrm{kHz}) \text {; } \\
\text { sound-treated rooms; Marantz } \\
\text { PMD670 portable; Sennheiser } \\
\text { M464-K6, 20cm from } \\
\text { participant's mouth. }\end{array}$ \\
\hline YorViS & $\begin{array}{l}18- \\
25\end{array}$ & $\begin{array}{l}\text { York } \\
\text { English }\end{array}$ & 21 & $\begin{array}{l}\text { Included: 1) Simulated } \\
\text { police interview, 2) } \\
\text { telephone call with } \\
\text { accomplice }\end{array}$ & $\begin{array}{l}\text { Studio quality (.wav, } 44.1 \mathrm{kHz} \text { ); } \\
\text { sound-treated rooms; Recorded } \\
\text { directly onto PC, via a TAC } \\
\text { Scorpion 16-8-2 mixing desk, } \\
\text { using an M-Audio 24/96 sound } \\
\text { card; Neumann U87Ai } \\
\text { condenser microphone (with } \\
\text { pop shield), } 25 \mathrm{~cm} \text { from } \\
\text { participant's mouth. Sennheiser } \\
\text { M464-K6, 20cm from } \\
\text { participant's mouth. }\end{array}$ \\
\hline WYRED & $\begin{array}{l}18- \\
30\end{array}$ & $\begin{array}{l}\text { West } \\
\text { Yorkshire } \\
\text { (Bradford, } \\
\text { Kirklees, } \\
\text { Wakefield) }\end{array}$ & $\begin{array}{l}180 \text { (60 for } \\
\text { each area) }\end{array}$ & $\begin{array}{l}\text { Included: 1) Simulated } \\
\text { police interview, 2) } \\
\text { telephone call with } \\
\text { accomplice }\end{array}$ & $\begin{array}{l}\text { Studio quality (.wav, } 44.1 \mathrm{kHz} \text { ) } \\
\text { sound-treated booths; PMD661 } \\
\text { KMII Handheld Solid State } \\
\text { Recorder; Sennheiser HSP 4, } \\
2 \mathrm{~cm} \text { from mouth }\end{array}$ \\
\hline
\end{tabular}

Table A2

Speaker selection ordered from closest Euclidean distance to farthest Euclidean distance from the target speaker, Experiment 1, nine-voice parade.

\begin{tabular}{ll}
\hline Database target group & Database speaker number \\
\hline DyViS 1 (SSBE) & $\mathbf{5 6}, 115,112,39, \underline{25}, 28,60,95,65,111 \mid 53,62,88,106,118$ \\
DyViS 2 (SSBE) & $\mathbf{2 3}, 87,2,50, \underline{76}, 4,37,31,35,32 \mid 1,11,21,47,113$ \\
DyViS 3 (SSBE) & $\mathbf{8 0}, 30,46,107, \underline{99}, 81,6,75,96,40 \mid 19,54,58,68,69$
\end{tabular}


WYRED 1 (Bradford)

WYRED 2 (Wakefield)

YorViS (York) $\mathbf{1 8 5}, 187,175,170, \underline{176}, 132,189,157,156,135 \mid 147,167$, $172,174,191$

166, $152,178,127, \underline{103}, 138,141,158,146,145 \mid 111,112$, $131,143,164$

$\mathbf{8}, 2,10,7, \underline{1}, 4,18,19,16,21 \mid 11,12,17,20,22$

Note. Target speakers are bolded and replacement speakers are underlined. Speakers listed after | represent discarded least-similar speakers.

\section{Table A3}

Speaker selection ordered from closest Euclidean distance to farthest Euclidean distance from the target speaker, Experiment 2, six-voice parade.

\begin{tabular}{ll}
\hline Database target group & Database speaker number \\
\hline DyViS 1 (SSBE) & $\mathbf{5 6}, 115,112,39, \underline{25}, 28,60 \mid 95,65,111,53,62,88,106,118$ \\
DyViS 2 (SSBE) & $\mathbf{2 3}, 87,2,50, \underline{76}, 4,37 \mid 31,35,32,1,11,21,47,113$ \\
DyViS 3 (SSBE) & $\mathbf{8 0}, 30,46,107, \underline{99}, 81,6 \mid 75,96,40,19,54,58,68,69$ \\
WYRED 1 (Bradford) & $\mathbf{1 8 5}, 187,175,170, \underline{176}, 132,189 \mid 157,156,135,147,167$, \\
& $172,174,191$ \\
WYRED 2 (Wakefield) & $\mathbf{1 6 6}, 152,178,127, \underline{103}, 138,141 \mid 158,146,145,111,112$, \\
& $131,143,164$ \\
YorViS (York) & $\mathbf{8}, 2,10,7, \underline{1}, 4,18 \mid 19,16,21,11,12,17,20,22$ \\
\hline
\end{tabular}

Note. Target speakers are bolded and replacement speakers are underlined. Speakers listed after | represent discarded least-similar speakers. 


\section{Appendix B (Experiment 1, nine-voice parade)}

\section{Table B1}

Model comparisons for accuracy data (Experiment 1, nine-voice parade).

\begin{tabular}{lcc}
\hline Model & $\Delta \widehat{\operatorname{elpd}}$ & $\Delta \mathrm{SE}$ \\
\hline Main effect: target presence & 0.0 & 0.0 \\
Main effects: sample duration + target presence & -1.5 & 0.8 \\
Interaction: sample duration x target presence & -2.1 & 1.3 \\
Intercept Only & -8.9 & 4.1 \\
Main effect: sample duration & -10.4 & 4.1 \\
\hline
\end{tabular}

Note. Models are ordered from the model with the highest predictive performance $\widehat{\operatorname{elpd}}$ (with standard error $[\mathrm{SE}])$ in the top row. $\Delta \widehat{\operatorname{elpd}}$ shows the difference in the predictive performance (with standard error $[\Delta \mathrm{SE}]$ ) of the best fitting model with main effect of target presence compared to all remaining models. All models were run with 30,000 iterations and model convergence was confirmed by the Rubin-Gelman statistic $(\hat{R}=1)($ Gelman \& Rubin, 1992). 


\section{Table B2}

Interaction model for accuracy data, 60s sample duration as reference (Experiment 1, nine-voice parade).

\begin{tabular}{lcllllll}
\hline Parameter & MAP & $95 \%$ HDI & $\hat{R}$ ESS & $\mathrm{BF}_{01}$ & $\mathrm{BF}_{10}$ & $\mathrm{p}$ (MAP) \\
\hline 15s Sample Duration & -0.23 & {$[-1.08-0.66]$} & 1 & 53679 & 2.01 & 0.50 & 0.907 \\
30s Sample Duration & -0.15 & {$[-1.01-0.73]$} & 1 & 55010 & 2.11 & 0.46 & 0.942 \\
Target-Present & 1.07 & {$[0.29-1.82]$} & 1 & 51850 & 0.06 & 14.44 & 0.027 \\
15s duration $\times$ Target Presence & 0.61 & {$[-0.46-1.61]$} & 1 & 49946 & 1.05 & 0.95 & 0.556 \\
30s duration $\times$ Target Presence & -0.02 & {$[-1.07-1.02]$} & 1 & 51341 & 1.9 & 0.53 & 0.999 \\
\hline
\end{tabular}

Note MAP = maximum a posteriori estimates (i.e., the mode of the posterior distribution); $95 \%$ $\mathrm{HDI}=95 \%$ Highest Density Interval; $\hat{R}=$ Rubin-Gelman statistic, $1=$ model convergence; ESS = effective sample size; $\mathrm{BF}=$ Bayes Factor calculated using the Save-Dickey density ratio method (Wagenmakers et al., 2010); $\mathrm{p}(\mathrm{MAP})=$ Bayesian equivalent of the p-value, related to the odds that a parameter has against the null hypothesis $\left(\mathrm{H}_{0}\right)$ (Makowski et al., 2019). 
Appendix C (Experiment 2, six-voice parade)

\section{Table C1}

Model comparisons for accuracy data (Experiment 2, six-voice parade).

\begin{tabular}{lcc}
\hline Model & $\Delta \widehat{\operatorname{elpd}}$ & $\Delta \mathrm{SE}$ \\
\hline Main effect: target presence & 0.0 & 0.0 \\
Main effects: sample duration + target presence & -1.4 & 1 \\
Interaction: sample duration x target presence & -2.4 & 1.1 \\
Intercept Only & -5.1 & 3.3 \\
Main effect: sample duration & -6.4 & 3.4 \\
\hline
\end{tabular}

Note. Models are ordered from the model with the highest predictive performance (with standard error [SE]) in the top row. $\Delta \widehat{e l p d}$ shows the difference in the predictive performance (with standard error $[\Delta \mathrm{SE}]$ ) of the best fitting model with main effect of target presence compared to all remaining models. All models were run with 30,000 iterations and model convergence was confirmed by the Rubin-Gelman statistic ( $\hat{R}=1)$ (Gelman \& Rubin, 1992). 


\section{Table C2}

Interaction model for accuracy data, 60s sample duration as reference (Experiment 2, six-voice parade).

\begin{tabular}{lccccccc}
\hline Parameter & MAP & 95\% HDI & & ESS & BF01 & BF10 & p (MAP) \\
\hline 15s Sample Duration & 0.02 & {$[-0.79-0.83]$} & 1 & 56806 & 2.46 & 0.412 & 0.996 \\
30s Sample Duration & -0.22 & {$[-1.08-0.59]$} & 1 & 57968 & 2.08 & 0.475 & 0.873 \\
Target-Present & 1.06 & {$[0.33-1.79]$} & 1 & 53689 & 0.04 & 23.90 & 0.015 \\
15s duration $\times$ Target Presence & -0.27 & {$[-1.28-0.72]$} & 1 & 53765 & 1.68 & 0.589 & 0.844 \\
30s duration $\times$ Target Presence & -0.20 & {$[-1.21-0.84]$} & 1 & 53757 & 1.8 & 0.563 & 0.938 \\
& & & & & & & \\
\hline
\end{tabular}

Note. $\mathrm{MAP}=$ maximum a posteriori estimates (i.e., the mode of the posterior distribution); $95 \%$

HDI $=95 \%$ Highest Density Interval; $\hat{R}=$ Rubin-Gelman statistic, $1=$ model convergence;

ESS = effective sample size; $\mathrm{BF}=$ Bayes Factor calculated using the Save-Dickey density ratio method (Wagenmakers et al., 2010); p (MAP) = Bayesian equivalent of the p-value, related to the odds that a parameter has against the null hypothesis $\left(\mathrm{H}_{0}\right)$ (Makowski et al., 2019). 


\section{Appendix D - Pooled analysis}

Data were analysed using Bayesian mixed models (Gelman et al., 2014; McElreath, 2016) with accurate parade identifications scored as 1 and inaccurate identifications as 0 in a 3 (sample duration: 15s, 30s, 60s) x 2 (target presence: present or absent) factorial design. This analysis treated the 6 targets as a random factor. The 60s sample duration condition was treated as the reference category. Leave-one-out cross validation was used to evaluate model comparisons (Vehtari et al., 2017). The fitted models' predictive performance was estimated as the sum of the expected log pointwise predictive density $(\widehat{\operatorname{elpd}})$ alongside its standard error (SE). A model with a difference in $\mathrm{SE}(\triangle \mathrm{SE})$ equal to or greater than 5 is suggestive of better predictive performance (Vehtari et al., 2017). As the interaction model had a $\Delta \mathrm{SE}$ of $0.7(<5)$ when compared to the target-presence only model (the model with the highest predictive capability), the interaction model was selected for inferential purposes.

\section{Table D1}

Model comparisons for accuracy data (Combined data).

\begin{tabular}{lll}
\hline Model & $\Delta \widehat{\operatorname{elpd}}$ & $\Delta \mathrm{SE}$ \\
\hline Main effect: Target-Present & 0 & 0 \\
All Main Effects & -1.2 & 1.2 \\
2-way Interactions & -1.3 & 0.7 \\
Null model & -14.6 & 5.3 \\
Main effect: Sample Duration & -15.8 & 5.4 \\
\hline
\end{tabular}


Note. Models are ordered from the model with the highest predictive performance (with standard error $[\mathrm{SE}])$ in the top row. $\Delta \widehat{\operatorname{elpd}}$ shows the difference in the predictive performance (with standard error $[\Delta \mathrm{SE}]$ ) of the best fitting model with main effect of target presence compared to all remaining models. All models were run with 30,000 iterations and model convergence was confirmed by the Rubin-Gelman statistic ( $\hat{R}=1)$ (Gelman \& Rubin, 1992).

\section{Table D2}

Interaction model for accuracy data, 60s sample duration as reference (combined data from Experiments 1 and 2).

Parameter MAP $\quad 95 \%$ HDI

$\hat{R} \quad$ ESS $\quad \mathrm{BF}_{01} \quad \mathrm{BF}_{10}$ $\mathrm{p}(\mathrm{MAP})$

\begin{tabular}{lccccccc}
\hline 15s Sample Duration & -0.07 & {$[-0.73-0.58]$} & 1 & 46133 & 2.91 & 0.344 & 0.987 \\
30s Sample Duration & -0.15 & {$[-0.82-0.52]$} & 1 & 50874 & 2.64 & 0.377 & 0.560 \\
Target Present & 1.10 & {$[0.52-1.69]$} & 1 & 49524 & $<.001$ & $>100$ & $<.001$ \\
15s duration $\times$ Target Presence & 0.14 & {$[-0.73-0.88]$} & 1 & 47197 & 2.36 & 0.422 & 0.844 \\
30s duration $\times$ Target Presence & -0.13 & {$[-1-0.64]$} & 1 & 46014 & 2.13 & 0.469 & 0.938 \\
\hline
\end{tabular}

Note. $\mathrm{MAP}=$ maximum a posteriori estimates (i.e., the mode of the posterior distribution); $95 \%$ HDI $=95 \%$ Highest Density Interval; $\hat{R}=$ Rubin-Gelman statistic, $1=$ model convergence; ESS = effective sample size; BF = Bayes Factor calculated using the Save-Dickey density ratio method (Wagenmakers et al., 2010); $\mathrm{p}(\mathrm{MAP})=$ Bayesian equivalent of the $\mathrm{p}$-value, related to the odds that a parameter has against the null hypothesis $\left(\mathrm{H}_{0}\right)$ (Makowski et al., 2019). 
Figure D1. Cell means with 95\% HDIs between speaker groups, combined data from Experiments 1 (nine voices) and 2 (six voices).

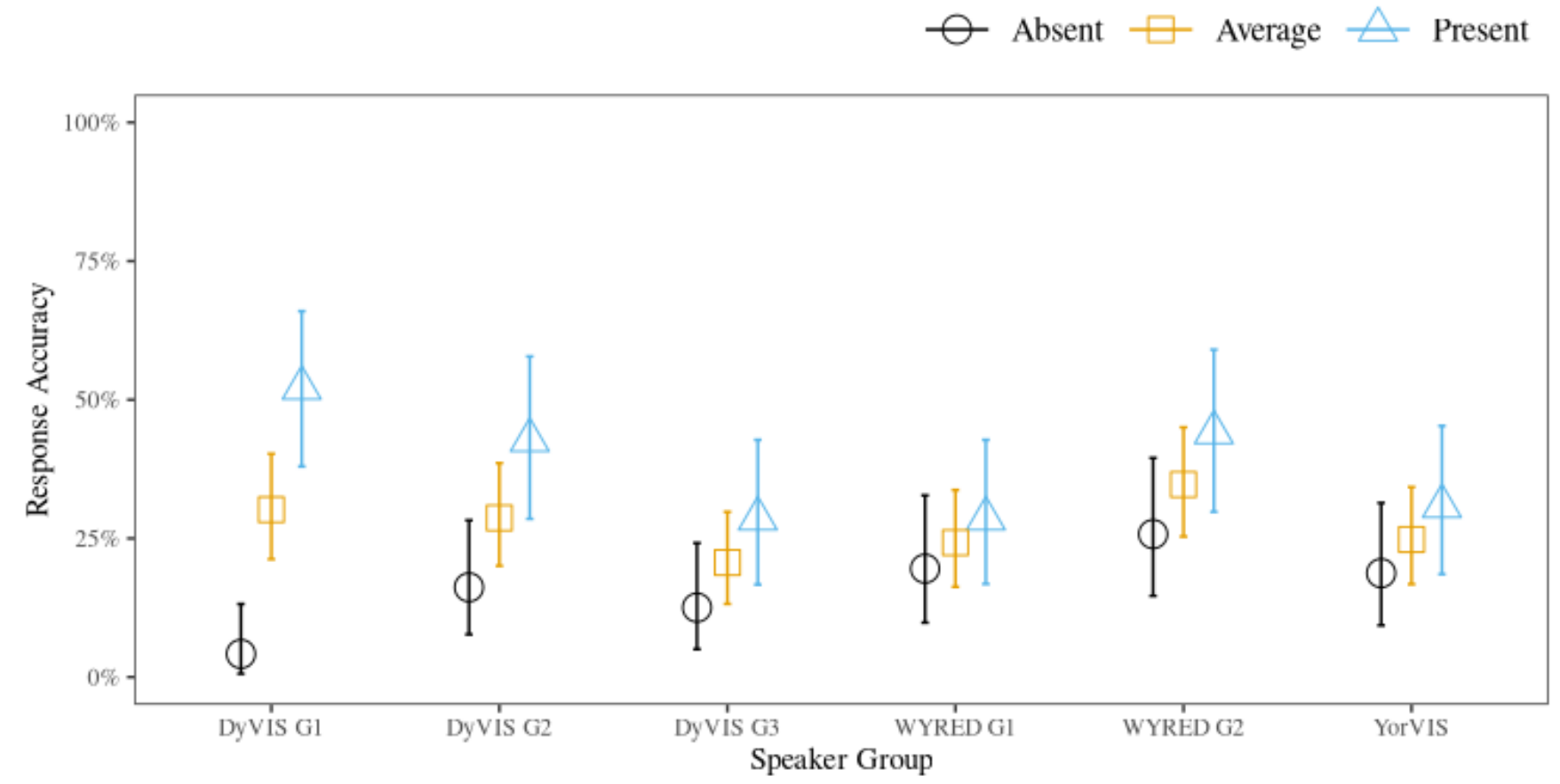

The cell means for by speaker-group binary accuracy displayed in Figure D1 illustrates relatively stable accuracy when collapsing across target presence. Modelling binary accuracy by speakergroup with DyViS Group 1 as the reference category, we found negligible evidence to support the hypothesis that meaningful differences were present: $D y V i S$ 2: $\hat{\beta}=-0.03$, HPDI: [-0.61 -0.57$]$, $\mathrm{BF}_{10}=0.302 ;$ DyViS 3: $\hat{\beta}=-0.42$, HPDI: [-1.07 - 0.17], $\mathrm{BF}_{10}=0.837 ;$ WYRED 1: $\hat{\beta}=-0.23$, HPDI: [-0.86 -0.35], $\mathrm{BF}_{10}=0.425 ;$ WYRED 2: $\hat{\beta}=0.23$, HPDI: $[-0.34-0.81], \mathrm{BF}_{10}$ $=0.403 ;$ YorViS: $\hat{\beta}=-0.21$, HPDI: [-0.82-0.38], $\mathrm{BF}_{10}=0.397$. 
Table D3

Model comparisons for accuracy data (Combined data, position effects).

\begin{tabular}{lll}
\hline Model & $\Delta \widehat{\operatorname{elpd}}$ & $\Delta \mathrm{SE}$ \\
\hline Main effect: Position & 0 & 0 \\
2-way interaction & -1.3 & 0.9 \\
Null model & -11.9 & 4.7 \\
Main effect: Parade Size & -12.9 & 4.7 \\
\hline
\end{tabular}

Note. Models are ordered from the model with the highest predictive performance (with standard error $[\mathrm{SE}]$ ) in the top row. $\Delta \widehat{\operatorname{elpd}}$ shows the difference in the predictive performance (with standard error $[\Delta \mathrm{SE}])$ of the best fitting model with main effect of target presence compared to all remaining models. All models were run with 30,000 iterations and model convergence was confirmed by the Rubin-Gelman statistic $(\hat{R}=1)$ (Gelman \& Rubin, 1992). 